\title{
Silicon-Rich Soil Amendments Impact Microbial Community Composition and The Composition of Arsm Bearing Microbes
}

\section{Gretchen E. Dykes}

University of Delaware

Matt A. Limmer

University of Delaware

Angelia L Seyfferth ( $\square$ angelias@udel.edu )

University of Delaware https://orcid.org/0000-0003-3589-6815

\section{Research Article}

Keywords: arsenic, methylation, rice, microbiome, silicon

Posted Date: March 11th, 2021

DOI: https://doi.org/10.21203/rs.3.rs-272042/v1

License: (c) (i) This work is licensed under a Creative Commons Attribution 4.0 International License.

Read Full License

Version of Record: A version of this preprint was published at Plant and Soil on August 26th, 2021. See the published version at https://doi.org/10.1007/s11104-021-05103-8. 


\section{Abstract}

Purpose: Arsenic (As) cycling in flooded rice paddies is driven by soil microbes which among other transformations can cause conversion between inorganic and organic As species. Silicon (Si)-rich soil amendments cause increased methylated As species, particularly DMA, in grain likely because they influence the microbial community responsible for As methylation, but the mechanism remains unclear.

Methods: To investigate how Si-rich amendments influenced the microbial community, we sequenced the 16S rRNA and arsM genes from rhizosphere soil collected at grain ripening from unamended rice paddy mesocosms or those amended with Si-rich rice husk, charred husk, or calcium silicate, and paired these data with geochemistry and As speciation in grain.

Results: We found that Si-amendments influenced the $16 \mathrm{~S}$ and arsM community composition. Increased C storage from calcium silicate amendment drove differences in the 16S community, whereas low redox from husk amendments drove differences in the arsM community. High grain DMA was not associated with treatment or microbial community, but with low redox.

Conclusions: Differences in grain As were observed independent of amendments, and did not correspond to differences in either the $16 \mathrm{~S}$ or arsM community. Instead, methane flux and redox correlated with differences in grain DMA, implying that methanogen activity and redox are more important factors than community composition in determining grain As speciation. Silicon amendments did not impact grain As, but impacted the microbial community composition, and the subset of arsM-bearing organisms. These findings imply that redox, porewater As, and methanogen activity are likely more important factors than arsM or overall microbial community composition in determining grain DMA levels.

\section{Introduction:}

Arsenic (As) cycling in flooded rice paddies impacts human health and is driven by soil microbes. Microbes are responsible for the release of As (iron-reducing bacteria), transformation of As between different oxidation states (As-reducing and oxidizing bacteria), and transformation of As between inorganic and organic forms (As-methylating and demethylating microbes) (Von Endt et al. 1968; Sanders 1979; Qin et al. 2006; Lomax et al. 2012; Yan et al. 2015). However, As-methylating microbes (AsMM) are poorly understood, and yet have a critical influence on grain As speciation.

Rice grain tends to contain higher concentrations of organic As species, mainly DMA, upon addition of silicon (Si)-rich soil amendments to paddy soil (Liu et al. 2014; Ma et al. 2014; Seyfferth et al. 2016; Teasley et al. 2017; Limmer et al. 2018; Yang et al. 2018). This implies that As methylation is promoted by Si-rich amendments but for reasons that are unresolved. A meta-analysis of Si-rich amendments found that increased grain organic As concentration was positively correlated with increased rice straw Si concentration (Seyfferth et al. 2018). For example, rice husk, charred husk, and calcium silicate all increase grain DMA compared to control, but likely for different reasons (Limmer et al. 2018; Limmer and Seyfferth 2020). Si-rich amendments can release inorganic As (Seyfferth and Fendorf 2012), increase 
organic matter, and/or decrease soil redox potential (Penido et al. 2016; Yang et al. 2018) depending on the type of amendment. While Si amendments promote As methylation and cause an increase in grain DMA concentration, they also can decrease grain inorganic As concentrations, which is more acutely toxic than DMA (Liu et al. 2014; Ma et al. 2014; Seyfferth et al. 2016, 2018, 2019a; Teasley et al. 2017; Limmer et al. 2018; Yang et al. 2018). Therefore, it is necessary to understand how Si amendments influence the soil microbial community involved in As cycling, especially As methylation.

Silicon-rich amendments may increase available inorganic As (iAs) rather than the less abundant DMA directly and consequently stimulate arsenic methylation (Dykes et al. 2020). Silicon-rich amendments increase the amount of porewater $\mathrm{Si}$, which in the circumneutral $\mathrm{pH}$ of flooded rice paddy porewaters is typically found as silicic acid. Silicic acid is a chemical analog of arsenous acid, the dominant As species in flooded rice paddy porewaters (Marin et al. 1993; Takahashi et al. 2004). Therefore, when Si amendments increase porewater $\mathrm{Si}$, increased competition with inorganic As for binding sites causes inorganic As desorption from soil solids (Luxton et al. 2006; Seyfferth and Fendorf 2012). This release of inorganic As increases the substrate for As methylation, and could therefore be responsible for promoting As methylation, resulting in higher levels of methylated As species in porewater and ultimately grain (Zhao et al. 2013b). However, the type of Si amendment affects this process, with some plant-based Si amendments such as rice husk or charred husk differing in Si dissolution rates compared to inorganic Si amendments (Teasley et al. 2017; Seyfferth et al. 2018; Limmer et al. 2018; Linam et al. 2021). This ultimately affects how much Si (and therefore As) is released to the porewater in a given period of time.

Incorporation of plant-based Si-rich amendments such as rice straw and rice husk also lead to an increase in soil organic matter and lower redox, which directly impacts the soil microbial community (Zhao et al. 2013a; Ma et al. 2014; Penido et al. 2016; Wang et al. 2019). Increased organic matter can drive lower redox potentials and increase As availability by increasing activity of anaerobic organisms that consume organic carbon such as methanogens and Fe and As reducing bacteria (Yang et al. 2018; Wang et al. 2019). Increased activity of Fe-reducers and As-reducers ultimately leads to increased inorganic As(III) in porewater (Yang et al. 2018; Wang et al. 2019) as adsorbed inorganic As is released from soil solids via reductive dissolution of Fe-(oxyhydr)oxides and $\mathrm{As}(\mathrm{V})$ is reduced to $\mathrm{As}(\mathrm{III})$, which is more mobile. Increasing inorganic As(III) leads to higher levels of porewater and grain DMA, most likely by increasing the amount of substrate (inorganic As) available to be methylated. Low redox and high organic matter can also enrich for an anaerobic microbial community, which may be important in As methylation (Zhao et al. 2013b). For example, methanogens were recently demonstrated to be important As methylators in low-redox environments (Viacava et al. 2020). Rice straw incorporation in particular enriched for mcrA/ mrtA, (a marker gene for methanogenesis) and increased dissolved $\mathrm{CH}_{4}$ concentrations and As release (Penido et al. 2016). However, straw and husk amendments behave differently as Si amendments (Penido et al. 2016), and the relationship between methanogens, methane flux, and grain organic As is still unclear.

To better elucidate how Si amendments influence the microbial community and the community of arsM harboring organisms, we sequenced the 16S rRNA gene and the arsM gene from rhizosphere soil at grain 
ripening from rice paddy mesocosms that were unamended (Control) or amended with rice husk (Husk), charred husk (Char), or calcium silicate (Silicate) and combined these analyses with extensive geochemical characterization that was previously described from the same field experiment (Limmer and Seyfferth 2020). While AsMM bearing the arsenic methyltransferase arsM gene have historically thought to have been responsible for transforming inorganic As into methylated species (Qin et al. 2006; Lomax et al. 2012), a growing body of literature suggests that in low redox environments, ars $M$ may not be active as an As detoxification strategy (Chen et al. 2019; Viacava et al. 2020). Instead, methanogens which methylate As through a non-specific side reaction of As may be responsible for As methylation (Thomas et al. 2011; Viacava et al. 2020). We therefore hypothesized that Si-amendments would impact redox and As availability, thereby indirectly impacting the microbial community. This work expands our understanding of how different soil Si-amendments impact the microbial community and our understanding of AsMM in rice paddies.

\section{Methods:}

\section{a. Field experiment}

DNA was extracted from isolated rice rhizospheres in year 2 after Si-rich amendments were added to soil in an outdoor mesocosm facility previously described (Limmer et al. 2018) where geochemical measurements were obtained over the growing season (Limmer and Seyfferth 2020). Briefly, paddy mesocosms $\left(2 \mathrm{~m}^{2}\right)$ were either unamended (Control) or amended with calcium silicate (Silicate), rice husk (Husk), or charred husk (Char) in triplicate at a rate of $5 \mathrm{Mg} \mathrm{Si}$ / ha prior to the 2015 growing season (Limmer et al. 2018). At the start of the 2016 growing season, each mesocosm was fertilized with urea and $\mathrm{KCl}$, but no additional Si amendments were applied. Each paddy was hand planted with 49 rice (Oryza sativa L. c.v. Jefferson) seedlings at the 3-4 leaf stage that had been germinated in field soil and topped with Cornell potting mix. At transplanting, five plants per paddy were contained within $100 \mu \mathrm{m}$ nylon mesh bags filled with paddy soil to define the rhizosphere for temporal sampling. Bags were 15.2 $\mathrm{cm} \times 31.8 \mathrm{~cm}$ for plants and rhizospheres sampled in this study.

\section{b. Microbial sampling}

To collect soil samples for microbial analysis, one bag containing one plant was pulled from each paddy at the grain ripening stage (88 days past transplant, (DPT)). Rhizosphere soil was collected by separating roots from the root mass and washing them in $25 \mathrm{~mL}$ of $18 \mathrm{M} \Omega$-cm sterile water, twice, by vortexing. Rhizosphere soils were frozen immediately following collection prior to DNA extraction. DNA was extracted from soils using the Qiagen PowerSoil DNA extraction kit, with modifications. From the beadbeating tubes, $200 \mu \mathrm{L}$ of powerbead solution was replaced with phenol:chloroform:isoamyl 25:24:1 alcohol at neutral $\mathrm{pH}$. The protocol was followed as written by the manufacturer until column binding. Here, equal parts lysate, solution $\mathrm{C} 4$, and absolute ethanol were homogenized before being loaded onto the column. Following this the DNA bound column was washed with $100 \%$ ethanol $(650 \mu \mathrm{L})$, then 
solution C5 $(500 \mu \mathrm{L})$. DNA was eluted with molecular biology grade water (based on personal communication with Qiagen representative).

\section{c. 16S rRNA gene sequencing and processing}

To assess the diversity of the whole microbial community, DNA samples were submitted to the Joint Genome Institute for paired end $(2 \times 300)$ Illumina MiSeq iTag sequencing of the $16 S$ rRNA gene. The V4V5 region was amplified using primers 515F-Y and 926R, according to Parada et al. 2015. 16S rRNA sequences were processed with the JGI pipeline. 16S rRNA gene sequences were deposited at JGI DOI $10.25585 / 1488298$. Briefly, sequences were de-multiplexed, quality-filtered, checked for chimeras, and clustered to $97 \%$ similarity with the iTagger pipeline (Tremblay et al. 2015). Processed sequences were imported into qiime2 (Bolyen et al. 2019), and filtered to only include sequences that occurred more than 10 times in the data set in at least 2 samples. Taxonomy was assigned to sequences using the sklearn naïve bayes feature classifier, trained with SILVA database version 132 (Quast et al. 2012).

\section{d. arsM gene sequencing and processing}

To assess the diversity of arsM-bearing organisms closest to the grain filling stage of rice growth, arsM genes were amplified and sequenced from rhizosphere samples collected at 88 DPT. DNA was extracted from soil samples for each paddy as described above, then amplified in a reaction with $5 \mathrm{ng}$ template DNA, $0.4 \mathrm{mM}$ barcoded forward and reverse primer, and 1X NEB Taq master mix in a PTC-100 thermocycler (MJ Research Inc.) for $10 \mathrm{~min}$ at $95^{\circ} \mathrm{C}$, followed by 30 cycles of $95^{\circ} \mathrm{C}$ for $30 \mathrm{~s}, 60^{\circ} \mathrm{C}$ for $45 \mathrm{~s}$, $72{ }^{\circ} \mathrm{C}$ for $60 \mathrm{~s}$, and a final incubation at $72{ }^{\circ} \mathrm{C}$ for 10 min. Barcoded primers were designed from PacBio ${ }^{\circledR}$ barcodes for SMRT ${ }^{\circledR}$ sequencing, based on arsM primers F1 and R2 from Zhao et al., 2013 (Table S1). Successful amplification for each sample was confirmed by gel electrophoresis. Amplicons were purified with MN PCR purification kit, according to manufacturer's instructions. Amplicon DNA concentration was determined using the Qubit BR DNA assay, according to manufacturer's instructions and $80 \mathrm{ng}$ of each barcoded amplicon were pooled and submitted for sequencing with PacBio RSII to the University of Delaware Genotyping and Sequencing Center.

arsM amplicons were initially filtered with the PacBio SMRT portal, including only sequences that had at least 5 circular consensus reads. Preliminarily filtered reads were imported into mothur (Schloss et al. 2009), and filtered to only include reads with an average quality score of at least $25,<1$ ambiguous bases, $<6$ homopolymers, minimum length of $200 \mathrm{bp}$, maximum length of $450 \mathrm{bp},<1$ difference in the primer, and $<1$ difference in the barcode. Sequences were aligned to a custom reference database, developed from the FunGene arsM database (described below) (Fish et al., 2013). Then, sequences were preclustered, merging groups within $2 \mathrm{nt}$ of each other, and chimeras were removed. Sequences were then clustered into groups with $99 \%$ similarity using the nearest neighbor method. Taxonomy was assigned to representative cluster sequences using BLAST against the NCBI nt database (2016), taking the top hit with best_hit_overhang parameter set to 0.25 , and best_hit_score_edge set to 0.05 . 
To develop a custom ars $M$ database for reference alignment, all ars $M$ sequences with a score above 50 were downloaded from the FunGene website, resulting in 23,043 sequences (Fish et al. 2013). For the reference nucleotide alignment, sequences were filtered with mothur (Schloss et al. 2009) to only include those with $<1$ ambiguous bases, $<10$ homopolymers, minimum length of $200 \mathrm{bp}$, maximum length of 500 $\mathrm{bp}$, and $<1$ difference in the primer for downstream analysis (3,795 sequences). For the reference alignment, sequences were de-replicated in mothur then aligned with MUSCLE (a widely-used, fast multiple sequence alignment software), then the alignment was refined using the muscle-refine option (Edgar 2004). The alignment was visually assessed using AliView (Larsson 2014).

To assess the range in diversity of our ars $M$ sequences against other ars $M$ proteins, we treed our sequences with reference proteins from NCBI. First, filtered unaligned arsM sequences from this study were translated and screened for frameshift sequencing errors using frameshift_polisher (https://github.com/dnasko/frameshift_polisher), implementing the 23,043 reference protein sequences downloaded from FunGene (described above) as a reference. Translated protein sequences were clustered at $90 \%$ similarity using CD-HIT (Fu et al. 2012), then aligned with MUSCLE. A separate reference protein sequence database, containing proteins between 200-400 bp from bacteria and archaea matching the search term "arsM" were downloaded from NCBI, along with eleven additional biochemically characterized arsenite methyltransferases, according to Reid et al. (2017). A new reference database was compiled for better comparison to previous literature. The NCBI reference sequences were aligned with MUSCLE, then the two alignments were aligned with each other using MUSCLE. The aligned set of arsM protein sequences was treed using RAxML-NG, using a single random starting tree and the JTT substitution model (Kozlov et al. 2019) and visualized using iTol ((Moore et al. 2017).

To investigate the diversity and enrichment patterns of arsM and 16S rRNA sequences in conjunction with chemical analysis, the arsM shared table (generated using mothur) and the $16 \mathrm{~S}$ biom table (generated using qiime2), along with associated rooted trees and taxonomy files were imported to $\mathrm{R}$ ( $\mathrm{R}$ Core Team 2019) using qiime2R (Bisanz 2018) and phyloseq (McMurdie and Holmes 2013). 16S rRNA gene sequences are deposited at JGI DOI 10.25585/1488298 and 16S rRNA gene sequences and arsM sequences are deposited at NCBI accession PRJNA690162.

\section{e. Chemical analysis}

After shoots and cleaned roots were collected, they were separated for chemical analysis of roots, shoots, husk, and grain. Husk, and grain were only analyzed at the last timepoint (98 DPT). After soil was cleaned from the root mass, roots were air dried and the iron plaque was removed using a cold dithionite-citratebicarbonate (DCB) extraction (Taylor and Crowder 1983). Plant parts were oven-dried and finely ground prior to microwave digestion with concentrated nitric acid (Seyfferth et al. 2016). After acid digestion, the samples were centrifuged and the acid fraction was decanted for ICP analysis. The remaining Si-rich precipitate was washed with DI water, centrifuged, and decanted three times prior to dissolving in $2 \mathrm{M}$ $\mathrm{NaOH}$ (Kraska and Breitenbeck 2010). The Si-rich fraction was analyzed using the molybdenum blue method. DCB extractions of root plaque and plant tissue acid digestions were subject to elemental 
analysis using ICP-OES (As, Al, B, Ca, Cu, Fe, K, Mg, Mn, P, Si, and Zn) or ICP-MS (As in grain, husk, straw, and root, Agilent 7500). In addition, grain As speciation was analyzed via HPLC-ICP-MS (Agilent 7500) after extraction in $2 \% \mathrm{HNO}_{3}$ (Maher et al. 2013) using a Hamilton PRP-100X column with $50 \mathrm{mM}$ ammonium carbonate mobile phase in $3 \%$ methanol (Jackson 2015). For acid digests, certified reference materials (NIST 1568a rice flour, WEPAL IPE 883 dianthus straw, and ERM CD281 rye grass) and method blanks were included in each digestion set. Average recoveries of target elements ranged from 92-113\% $(\mathrm{n}=10-15)$. For grain As speciation analysis, NIST 1568b rice flour was analyzed and recovery of inorganic As and DMA, the primary As species, were $102 \%$ and $95 \%$, respectively.

Porewater via rhizon and methane flux were monitored weekly throughout the experiment as described by Limmer and Seyfferth, 2020. Porewater $\mathrm{pH}$ and redox were measured by electrodes immediately after sampling. Si and Fe(II) were measured colorimetrically with the molybdenum blue (Kraska and Breitenbeck 2010) and ferrozine methods, respectively (Stookey 1970). Finally, Al, B, Ca, Cu, Mg, Mn, Fe, K, $\mathrm{Na}, \mathrm{P}, \mathrm{S}$, and $\mathrm{Zn}$ were measured after acidification (2\% HNO3) via ICP-OES and total As was measured by ICP-MS (Agilent 7500). The As species MMA, DMA, and inorganic As were measured via HPLC-ICP-MS using the same instrumental setup as for grain As speciation described above. Because samples were preserved with nitric acid and stored under oxic conditions, $\mathrm{As}(\mathrm{III})$ and $\mathrm{As}(\mathrm{V})$ were summed and are reported as inorganic As. DOC was measured by vario TOC cube (Elementar). Methane flux was also measured on a weekly basis according to Limmer and Seyfferth (2020). Because porewater between 5589 DPT was most predictive of mature grain As concentrations (Limmer and Seyfferth 2020), the median porewater or median methane flux value in this range of sampling was used for analysis with microbial data. Following the 2016 growing season, five soil cores from 0-10 cm depth were collected in diagonal pattern across each rice paddy and composited for analysis of solid-phase As pools by As sequential extraction, as described in detail by (Seyfferth et al. 2019b).The extractants were $0.05 \mathrm{M}$ ammonium sulfate for fraction F1 (non-specifically sorbed As), $0.05 \mathrm{M}$ ammonium phosphate for fraction F2 (specifically sorbed As), $0.2 \mathrm{M}$ acid ammonium oxalate for fraction F3 (As associated with amorphous Fe and Al oxides), $0.2 \mathrm{M}$ ammonium oxalate and ascorbic acid for fraction F4 (As associated with crystalline $\mathrm{Fe} / \mathrm{Al}$ oxides) and nitric acid and hydrogen peroxide for fraction F5 (residual As) (Motuzova et al. 2006).

\section{f. Statistical analysis}

All statistical analyses were performed in $\mathrm{R}$ and are noted where described. Here, we compared the median porewater values between 55-89 DPT for each paddy to alpha-diversity parameters and relative abundance of selected OTUs using Spearman's rank correlation. A Kruskal-Wallis test was used for comparisons between treatment groups and grain DMA groups, with Dunn's post hoc test. The ADONIS test was used to compared beta-diversity across treatment groups and grain DMA categories. The envfit function included in the R package vegan (Oksanen et al. 2019) was used for comparisons of betadiversity with continuous variables.

\section{Results:}


We sequenced the $16 \mathrm{~S}$ rRNA and ars $M$ genes from the rhizosphere soils collected at grain ripening (88 DPT) of 12 rice paddy mesocosms amended with husk, char, silicate, or unamended control and paired these data with geochemical measurements from the rice paddies that were previously described (Seyfferth et al. 2019b; Limmer and Seyfferth 2020). We obtained 36,829 raw arsM sequences (with at least 5 circular consensus reads) from PacBio sequencing, of which 24,627 (67\%) remained after quality filtering. Reads were clustered into 4,638 OTUs at $99 \%$ similarity. The mean sequence length was $302 \mathrm{bp}$. We obtained a moderate sequencing depth of ars $M$ sequences, as determined by the ars $M$ rarefaction curve (Fig. S1). arsM sequencing effort are summarized by sample in Table S2. For 16S rRNA gene sequencing, we obtained 7,368,144 raw reads from Illumina sequencing, of which 2,574,812 (35\%) remained after quality filtering. Reads were clustered at $97 \%$ similarity into 11,678 OTUs with an average length of 253 base pairs. We obtained a good sequencing depth of $16 \mathrm{~S}$ sequences, as determined by the 16S rarefaction curve (Fig. S2). 16S rRNA gene sequencing effort are summarized by sample in Table S3.

\section{a. Treatment and $16 \mathrm{~S}$ diversity}

The 16S Shannon diversity and Pielou's evenness in the rhizosphere at ripening was significantly higher in the husk-amended soils than control or silicate-amended soils (Fig. 1a and b, pairwise Kruskal-Wallis, $\mathrm{p}$ <0.05). $16 \mathrm{~S}$ alpha-diversity did not significantly correlate with any porewater chemistry factors, methane flux, grain As, or solid phase As pools (Supplemental file Table 1).

The $16 \mathrm{~S}$ rhizosphere community composition at ripening differed by treatment, with treatment explaining $54 \%$ of the community variation (Fig. $1 c, A D O N I S, R^{2}=0.54, p=0.015$ ). The $16 S$ community of control and char amended paddies were similar to each other, with husk and silicate amended paddies separate from each other and from control and char-amended soils, which clustered together (Fig. 1c). The 16S community at grain ripening in the rhizosphere was dominated by Sideroxydans OTU2, Spirochaetaceae OTU3, and Prolixibacteraceae BSV13 OTU8, which were the three most abundant OTUs across all 12 paddies (Fig. 2). To determine OTUs that were indicator species for each treatment, we used the multilevel pattern analysis function with the $\mathrm{R}$ indicspecies package. Several OTUs were identified as indicators of different treatments; 201 were indicators of silicate, 87 were indicators of husk, 35 were indicators of char, and 27 were indicators of control (Fig. S3-S6). Of all the indicator OTUs, most were uncultured, unclassified, or heterotrophs (Fig 3, Supplemental file table 2).

Differences in Si amendments correlated with differences in the soil microbial community composition (Fig. 4, Supplemental file table 3). Silicate-amended paddies led to high porewater $\mathrm{DOC}, \mathrm{Ca}$, and $\mathrm{Mg}$ concentrations, and we observed that differences in 16S community composition correlated with porewater DOC (Vegan envfit function, $R 2=0.56, p=0.05)$ along with $\mathrm{Ca}\left(\mathrm{R}^{2}=0.56, \mathrm{p}=0.02\right)$ and $\mathrm{Mg}$ $\left(R^{2}=0.52, p=0.04\right)$, which were covariates with DOC (Fig. 4, Supplemental file table 3 ). In addition, divalent cations in the root (which are a proxy of chemical species available to the root) such as $\mathrm{Ca}\left(\mathrm{R}^{2}=0.56\right.$, $p=0.03), M g\left(R^{2}=0.53, p=0.05\right)$, and $M n\left(R^{2}=0.44, p=0.07\right)$ and shoot $M n\left(R^{2}=0.59, p=0.03\right)$ were significantly correlated with differences in $16 \mathrm{~S}$ community composition, with a high concentration of divalent cations associated with silicate amended paddies (Supplemental file table 3). Indicator species 
associated with silicate amended soils positively correlated with porewater DOC (Supplemental file table 4) and included organotrophs such as Geobacter and Gemmatimonadaceae.

Because Fe-reducing bacteria (FeRB) and methanogens have previously been implicated as important players in As cycling and DOC consumption in wetland soils, we compared the sum relative abundance of organisms identified as methanogens (OTUs listed in Fig. S8) or FeRB (OTUs listed in Fig. S9) by taxonomy and their relationship with each other and geochemical factors. The relative abundance of methanogens and FeRB were significantly different across treatments, (Fig. 5a,b Kruskal-Wallis methanogens $p=0.10$, FeRB $p=0.08$ ) with methanogens highest in the husk treatment and FeRB lowest in the husk treatment.

\section{b. Treatment and arsM diversity}

The arsM OTUs were widely distributed amongst a set of representative sequences, with some clustering with previously observed ars $M$ sequences but many clustering independently of previously observed arsM sequences (Fig. S9). This indicates that our arsM sequences expand upon the previously observed diversity in arsM sequences. Of the 165 OTUs that were assigned taxonomy with the NCBI blast database (3.6\% of total OTUs), 55 were designated Rhodopseudomonas palustris, the organism from which arsM was originally characterized as an As-detoxifying As-methylating protein coding gene (Supplemental file table 5) (Qin et al. 2006). Rhodopseudomonas palustris OTUs were found in all samples. Other arsM OTUs included Gemmatirosa kalamazooensis (10 OTUs), Planctomycetes (22 OTUs), Rubrivivax gelatinosus (6 OTUs), Sphaerobacter thermophilus (9 OTUs), Stenotrophomonas sp. NA06056 (8 OTUs), and various Streptomyces species (11 OTUs). Four OTUs ( $0.09 \%$ of OTUs) were classified as archaea in the families Haloarculaceae and Halobacteriaceae.

The Shannon diversity and Pielou's evenness of arsM-bearing organisms in the rhizosphere at ripening generally did not differ between treatments; however, silicate-amended soils had higher Shannon diversity than char-amended soils (Fig 6a,b, pairwise Kruskal-Wallis, $\mathrm{p}<0.05$ ). In addition, Shannon diversity was positively correlated with porewater $\mathrm{Mg}$ (Spearman's rank correlation, rho $=0.60, \mathrm{p}=0.04$ ) and $\mathrm{DOC}$ (rho $=0.57, p=0.06)$, and negatively correlated with porewater $B(r h o=-0.70, p=0.02)$ and fraction $F 3$ As at harvest (associated with amorphous and Fe and aluminum oxides, rho $=-0.70, p=0.01$, Supplemental file table 1). Pielou's evenness was positively correlated with methane flux (rho $=0.64, p=0.03$ ), grain inorganic As at harvest ( $r h o=0.62, p=0.03$ ), and negatively correlated with fraction $F 2$ As at harvest (specifically sorbed As pool, rho $=-0.64, p=0.03$ ), fraction $F 3$ As at harvest (rho $=-0.63, p=0.03)$, and $B($ rho $=-0.59, p=0.04$, Supplemental file table 1).

Similar to the $16 \mathrm{~S}$ community, the distribution of rhizosphere arsM differed by treatment at ripening (Fig. $6 c$, ADONIS of Bray-Curtis dissimilarity $p<0.07$, homogeneity of dispersion $p>0.05$ ). In the CCA, control and char-amended paddies clustered more closely to each other, with husk- and silicate-amended paddies distinct from other treatments. Treatment explained $61 \%$ of the variation in the ars $M$ community (ADONIS, $R^{2}=0.61, p=0.034$ ). Of the thirty most abundant arsM OTUs in the rhizosphere at grain ripening, 
only one was identified beyond the kingdom level as Rhodopseudomonas palustris (Fig. 7). Twenty-four OTUs were identified as indicators of husk amended paddies, compared to one indicator of char, six indicators of silicate, and three indicators of control (Fig. 8). Hierarchical clustering of paddies considering just indicator OTUs only grouped husk-amended paddies together (Fig. S10). No indicator OTUs were assigned taxonomy more detailed than the kingdom level.

The arsM community composition correlated with treatment-induced differences in geochemical parameters (Fig. 9). Silicate-amended paddies were associated with higher porewater $\mathrm{Ca}$, and the distribution of arsM correlated with porewater $\mathrm{Ca}$ (envfit, $\mathrm{R}^{2}=0.52, \mathrm{p}=0.05$, Supplemental file table 6 ). Other plant nutrients like $\mathrm{Cu}\left(R^{2}=0.58, p=0.03\right), \mathrm{Mg}\left(R^{2}=0.45, p=0.1\right)$ and $P\left(R^{2}=0.51, p=0.06\right)$ were also correlated with the distribution of ars $M$ and were associated with silicate-amended paddies. In contrast, husk-amended paddies were associated with higher $\mathrm{CH}_{4}$ fluxes than other treatments, and arsM distribution also correlated with $\mathrm{CH}_{4}$ flux (envfit, $\mathrm{R}^{2}=0.75, \mathrm{p}=0.004$, Fig. 9). In addition, porewater $\mathrm{pH}$ $\left(R^{2}=0.56, p=0.04\right), F e(I I)\left(R^{2}=0.56, p=0.03\right)$, and inorganic $A s\left(R^{2}=0.44, p=0.08\right)$ correlated with ars $M$ distribution, and were covariates with methane flux. Furthermore, husk indicator OTUs were positively correlated methane flux, Fe(II), porewater inorganic As, and with each other (Fig. S11).

\section{c. Grain As and microbial communities}

Although grain DMA did not differ by treatments, a subset of paddies that were spatially grouped and had low soil redox potentials had high grain DMA concentrations (Fig. 10a,b); of these, two were huskamended, one was silicate-amended and one was from the unamended control treatment. To probe this further, we compared the microbial community to grain DMA levels and to porewater geochemistry to better understand biogeochemical factors contributing to high grain DMA. The arsM distribution did not correlate with grain As speciation (when treated continuously, envfit, $p>0.05$,) or any soil As fraction ( $p>0.05$, envfit, Supplemental file table 6). Furthermore, the distribution of ars $M$ did not differ between grain DMA categories (when treated categorically, Fig. 10c, $A D O N I S, R^{2}=0.08, p=0.7$ ). In addition, Shannon diversity and Pielou's evenness of arsM was not different by grain DMA categories (KruskalWallis, $p>0.05$, Fig. 10e,f). Only Pielou's evenness was positively correlated with grain inorganic As (Supplemental file table 1, Spearman's rank correlation, rho $=0.63, p=0.04$ ), however no arsM alpha diversity factors were related to grain DMA.

Similar to arsM, the distribution of the $16 \mathrm{~S}$ microbial community did not differ between grain DMA categories (Fig. 10d, ADONIS, $\mathrm{R}^{2}=0.09, \mathrm{p}=0.5$ ), and also did not correlate with grain As (when treated continuously, envfit, $p>0.05$, Supplemental file table 3 ). $16 \mathrm{~S}$ alpha diversity parameters (Shannon diversity, Pielou's evenness) did not differ by DMA category or correlate with grain As (Fig. 10g,h, Supplemental file table 1, Kruskal-Wallis, $p>0.05$ ).

We also specifically examined sum relative abundance of methanogens and FeRB from the 16S rRNA sequencing dataset, and although FeRB were slightly higher in paddies in the low grain DMA category, neither microbial functional group was significantly different between high and low grain DMA paddies 
(Fig. 11a,b, Kruskal-Wallis p>0.1). However, we did find that median methane flux was negatively correlated with the relative abundance of FeRB (Fig. 11C, Spearman's rank correlation, rho $=-0.67, p=0.02$ ) and median methane flux was positively correlated with grain DMA (Fig. 11d, Spearman's rank correlation, rho $=0.55, p=0.07$ ).

Finally, to investigate the geochemical relationships that may have led to high grain DMA in the spatiallygrouped paddies (Fig. 10a, b) we compared grain DMA with various porewater factors. We found that grain DMA was positively correlated with median porewater DMA (Fig. 12a, Spearman's rank correlation, rho $=0.65, p=0.02$ ) and median porewater As (Fig. 12d, Spearman's rank correlation, rho=0.74, $p=0.008$ ). Median porewater As and grain DMA were both negatively correlated with median porewater redox potential (porewater As Fig. 12b, Spearman's rank correlation, rho $=-0.74, p=0.008$; grain DMA Fig. 12c, Spearman's rank correlation, rho $=-0.59, \mathrm{p}=0.05)$.

\section{Discussion:}

Different types of soil Si amendments have been explored as a strategy to decrease inorganic As concentrations in rice grain (Liu et al. 2014; Ma et al. 2014; Seyfferth et al. 2016; Teasley et al. 2017; Limmer et al. 2018; Yang et al. 2018) but their impact on the soil microbial community has not been fully explored. Si-rich amendments decrease grain inorganic As concentrations and promote soil production and plant uptake of DMA, but they also affect other important geochemistry like plant nutrition and methane emissions (Liu et al. 2014; Ma et al. 2014; Seyfferth et al. 2016; Teasley et al. 2017; Limmer et al. 2018; Yang et al. 2018). Here, we investigated the influence of Si-rich amendments on the 16S and ars $M$ microbial communities, and how these communities influenced grain As speciation by sequencing $16 S$ rRNA and arsM genes from the rhizosphere of rice plants at the grain ripening stage. It has previously been suggested that Si amendments would indirectly influence the microbial community, for example by As desorption from increased competition with Si for binding sites on soil solids (Seyfferth and Fendorf 2012; Dykes et al. 2020) and increasing substrate for inorganic As methylation. We hypothesized that Siamendments would indirectly impact the microbial community by impacting redox and As availability. We found that Si-rich amendments caused geochemical differences that corresponded with change in both the $16 \mathrm{~S}$ and ars $M$ community composition.

The study described here provides a microbial analysis of an experiment described by Limmer and Seyfferth (2020) who reported that after two years of growth in soil that was treated with Si-rich amendments at the beginning of year 1, rice plants continued to have higher Si levels in Si-amended paddies compared to non-amended control paddies regardless of the type of Si amendment. That study also found that husk amendment increased methane emissions whereas silicate amendment increased porewater DOC and some divalent cations. Here, we paired these findings with analysis of the microbial community composition, which was hypothesized to be influenced by amendment-driven changes in geochemistry (Limmer and Seyfferth 2020). We found that treatment with calcium silicate led to higher porewater levels of DOC and divalent cations, whereas treatment with Si-rich rice husk led to lower porewater Fe(II) and inorganic As, both of which shaped the microbial community. Regardless of the 
effect treatments had on shaping the microbial community, grain DMA levels were not controlled by treatment but were driven by over-arching differences in redox-sensitive variables.

\section{a. Treatment effects on geochemistry and the microbial community}

Silicate-amended paddies may have impacted the microbial community by influencing $\mathrm{C}$ storage in year one growth. In silicate amended paddies, where Ca was elevated due to the addition of Si as calcium silicate, Ca could have facilitated DOC binding to Fe/Mn oxides via Fe(or Mn)-Ca-DOC ternary complexes (Sowers et al. 2018b). In year two, as redox continued to decrease from year one (Limmer and Seyfferth 2020), reductive dissolution of $\mathrm{Fe}$ and $\mathrm{Mn}$ oxides would release $\mathrm{Mn}, \mathrm{Fe}$, and As into solution, and in $\mathrm{Ca}$ silicate amended paddies reductive dissolution could additionally release stored DOC and Ca. Mg has additionally been hypothesized to facilitate DOC binding to Fe/Mn oxides and therefore, like $\mathrm{Ca}$, could be released with reductive dissolution of Fe/Mn oxides (Sowers et al. 2018a).

Increased DOC, driven by silicate treatment, was correlated with differences in $16 \mathrm{~S}$ community composition, and was associated with several organoheterotrophic microorganisms (Fig. 1, 3, Supplemental file table 2). In addition, most $16 \mathrm{~S}$ silicate treatment indicator OTUs were organoheterotrophic. In particular, organisms in the family Gemmatimonadaceae were often identified as indicator species, with one of the most abundant Gemmatimonadace OTUs (OTU170) slightly enriched in the silicate treatment. Gemmatimonadaceae have previously been identified as possible AsMM (Jia et al. 2013), but no Gemmatimonadaceae were identified in our arsM sequencing data set (Supplemental file table 5). Members of Gemmatimonadaceae have previously been identified as poly-phosphate accumulating organisms (Zhang et al.) facultative photoheterotrophs (Zeng et al. 2015), and $\mathrm{N}_{2} \mathrm{O}$ reducing organisms under oxygen starvation (Park et al. 2017). The versatility of this family combined with its organic carbon consumption could be why these organisms were enriched in the silicate treatment. In addition, several Geobacter OTUs were impacted by Si amendments, with silicate treatment enriching for Geobacter OTU6 and OTU66 (Fig. 3). Geobacter are classified within the deltaproteobacteria, which have previously been associated with AsMM (Jia et al. 2013), but likely also influence grain As by increasing porewater As availability through reductive dissolution of $\mathrm{Fe} / \mathrm{Mn}$ (oxy)hydroxides (Yang et al. 2018; Wang et al. 2019). Geobacter are chemoorganotrophs known for their role in Fe(III) and $\mathrm{Mn}$ (IV) reduction (Lovley et al. 2011). The high abundance of DOC and $\mathrm{Mn}$ in silicate amended paddies could explain why several Geobacter OTUs were silicate treatment indicators.

Because DOC drove differences in the microbial community composition, and DOC is a critical substrate for FeRB and methanogens, we chose to focus on how treatments influenced these microbial groups, and additionally how these groups were related to grain As speciation. Both groups are organisms that can influence the availability and speciation of As in paddy porewater. Methanogens were enriched in the husk treatment, whereas FeRB were lowest in the husk treatment, and methane flux and FeRB relative abundance were negatively correlated (Fig. 5a,b, 11c). Previous work has suggested that an active FeRB community can suppress methanogenic activity through competition for organic $\mathrm{C}$ (Roden and Wetzel 1996; Weiss et al. 2007) in accordance with our findings here. Neither methanogens nor FeRB as 
functional groups were enriched in the silicate amended paddies, which had the highest DOC (Fig. 5a,b). While it is also possible that the quality of DOC or sorbed $\mathrm{C}$ influenced the microbial community composition, we did not characterize DOC beyond porewater concentration here, but should be further explored. In addition, we found that methane flux was positively correlated with grain DMA at harvest (Fig. 11d). This suggests, in accordance with recent findings (Viacava et al. 2020), that methanogens may be important As-methylaters independent of arsM in low-redox environments.

While increasingly stronger reducing conditions from year one to year two influenced all paddies, the husk paddies in particular had low average redox that drove differences in the AsMM community (Fig. 9). Husk-amended paddies were associated with higher methane flux, inorganic As, and Fe(II); all factors that were correlated with differences in arsM community composition (Fig. 9). This is similar to previous work in which $\mathrm{Fe}, \mathrm{NO}_{3}{ }^{-}$, and total As (among other factors) were associated with AsMM community composition (Zhang et al. 2015). $\mathrm{Fe}, \mathrm{NO}_{3}{ }^{-}$, and As are redox-sensitive elements and therefore support our interpretation that redox impacts the community composition of AsMM. Redox may impact the community of AsMM by enriching for anaerobic organisms and/or by increasing As availability (Zhao et al. 2013b).

\section{b. Redox effects, grain As, and the microbial community}

Previous studies have reported the increase of organic As in grain (Ma et al. 2014; Seyfferth et al. 2016; Limmer et al. 2018; Yang et al. 2018) and porewater (Huang et al., 2012; Jia et al., 2012) with soil amendments such as rice straw, rice husk, dried distillers grain, and clover, but here husk treatment did not correspond to significantly higher grain DMA than control (Limmer and Seyfferth 2020). Rather, the paddies with the highest grain DMA were clustered spatially in field location (Fig. 10) and belonged to control, husk, and silicate treatments. This allowed us to examine the ars $M$ and $16 \mathrm{~S}$ microbial communities between high and low grain DMA paddies. Community composition of either arsM or the $16 \mathrm{~S}$ community did not correlate with differences in grain DMA (Fig. 10c,d). We also did not observe differences between paddies with different grain DMA levels within paddies of the same treatment amongst the 30 most abundant arsM or 16S OTUs (by median relative abundance in rhizosphere at grain ripening, Fig. 2,7) or amongst methanogens or FeRB (Fig. S7, S8). While we did not observe differences in the microbial community composition between high grain DMA and low grain DMA rhizosphere soils, there could be differences in arsM activity, or even cryptic cycling of methylated As species between AsMM and As de-methylating microbes, but neither were able to be measured in this study. On the other hand, redox was negatively correlated with grain DMA (Fig. 12c), which supports that redox and As availability were more important than arsM or $16 \mathrm{~S}$ microbial community composition in influencing grain DMA levels. In addition, methane flux was positively correlated with grain DMA which implies that methanogen activity relates to grain DMA levels (Fig. 11d).

Redox factors, including porewater inorganic As, but not grain DMA, corresponded with differences in the ars $\mathrm{M}$ microbial community composition, primarily separating the husk-amended community from other treatments (Fig. 8). This was surprising, given that redox was negatively correlated with grain DMA (Fig. 
9c). Of the three husk paddies, paddy $7 \& 8$ had high grain DMA and low redox, but paddy 5 had low redox and low grain DMA (Fig. 10ab,12). Low redox and low grain DMA were also observed in paddy 10 (control). This implies that low redox from husk amendment influences the community composition of ars $M$, but the change in ars $M$ community composition from husk amendment is not responsible for increased grain DMA. Recently Viacava et al. (2020) found that anaerobic microbes carrying ars $M$ did not express arsM in response to elevated As(III), but aerobic organisms did. Instead, the only anaerobic organism that demonstrated the capacity for As-methylation was a methanogen, possibly methylating As through side reactions with methyltransferase mtaA (Thomas et al. 2011; Viacava et al. 2020). Anaerobes may not methylate As for detoxification under low redox conditions due to increased toxicity of trivalent methylated As products compared to inorganic As, and instead could use arsM in microbial chemical warfare (Chen et al. 2019). This is in accordance with our finding that redox influenced arsM community composition (Fig. 8). Viacava et al. (2020) also suggested that when the As(III)-efflux pump acr3 was active, little As(III) was able to accumulate in the cell and be methylated. Perhaps under low-redox conditions organisms with active As(III) efflux pumps are selected for and methanogens methylate As independent of ars $M$, while under higher redox conditions (where less-toxic pentavalent methylated As species persist) As-methylation via arsM is more active. In this scenario, increased porewater As under low redox conditions would also result in higher grain DMA, as it would still increase the substrate for non-specific methylation by methanogens. We also found grain DMA did not correlate with arsM Shannon diversity (Supplemental file table 1). This is consistent with previous work in acid mine drainage sites where ars $M$ was widely distributed, and ars $M$ distribution and richness did not follow the As pollution gradient (Desoeuvre et al. 2016). Together, these findings indicate that redox-induced changes in ars $M$ community composition are not responsible for observed differences in grain DMA. We hypothesize in paddies with low redox and low grain DMA (i.e. paddies 5 and 10, Fig. 10,12) methanogens were less active than in paddies with low redox and high grain DMA. This is supported by our observation that methane flux was positively correlated with grain DMA.

\section{Conclusions:}

We found that Si-amendments influenced both the whole microbial community and the As-methylating subset of the microbial community. Increases in $\mathrm{Ca}$ from calcium silicate treatments could have promoted $\mathrm{C}$ storage in year one, resulting in $\mathrm{C}$ release in year two, which may have driven changes in both 16S rRNA and arsM gene distribution. In addition, low redox due to husk amendment may have driven changes in ars $M$ community composition. Neither the $16 \mathrm{~S}$ community nor the ars $M$ community composition was associated with differences in grain DMA levels; however, methane flux, porewater redox potential, and porewater As were correlated with grain DMA. These findings imply that redox, porewater As, and methanogen activity are likely more important factors than ars $M$ or overall microbial community composition in determining grain DMA levels. Future work investigating the activity of methanogens in relation to grain DMA is warranted.

\section{Declarations:}


Acknowledgements:

The authors thank Clara Chan, for research discussions, Brewster Kingham and Shawn Polson for arsM sequencing design, and Patrick Wise, Kristy Northrup, Weida Wu, Ayofela Dare, Kendall McCoach, Fred Teasley, Douglas Amaral, Ruifang Hu, Alesia Hunter, Julia O’Brien and Heather Eby for field assistance.

\section{Funding sources:}

This work was supported by the National Science Foundation Grant No. 1350580, USDA NIFA Grant Nos. 2016-67013-24846 and 2018-67019-27796 to A.L.S.; the DENIN Environmental Fellows program, the University of Delaware Doctoral and Dissertation Fellowships, and the Preston C. Townsend Biotechnology Fellowship to G.E.D. Sequencing for this project was completed by the Joint Genome Institute for project ID 503349.

Conflicts of interest: The authors declare no conflicts of interest.

Data availability: 16S rRNA gene sequences are deposited at JGI DOI 10.25585/1488298 and 16S rRNA gene sequences and arsM sequences are deposited at NCBI accession PRJNA690162.

Code availability: not applicable

\section{References:}

Bisanz JE (2018) qiime2R: Importing QIIME2 artifacts and associated data into R sessions

Bolyen E, Rideout JR, Dillon MR, et al (2019) Reproducible, interactive, scalable and extensible microbiome data science using QIIME 2. Nat Biotechnol 37:852-857. https://doi.org/10.1038/s41587019-0209-9

Chen J, Yoshinaga M, Rosen BP (2019) The antibiotic action of methylarsenite is an emergent property of microbial communities: MAs(III) is an antibiotic. Mol Microbiol 111:487-494.

https://doi.org/10.1111/mmi.14169

Desoeuvre A, Casiot C, Héry M (2016) Diversity and Distribution of Arsenic-Related Genes Along a Pollution Gradient in a River Affected by Acid Mine Drainage. Microb Ecol 71:672-685.

https://doi.org/10.1007/s00248-015-0710-8

Dykes GE, Chari NR, Seyfferth AL (2020) Si-induced DMA desorption is not the driver for enhanced DMA availability after $\mathrm{Si}$ addition to flooded soils. Science of The Total Environment 739:139906.

https://doi.org/10.1016/j.scitotenv.2020.139906

Edgar RC (2004) MUSCLE: multiple sequence alignment with high accuracy and high throughput. Nucleic Acids Research 32:1792-1797. https://doi.org/10.1093/nar/gkh340 
Fish JA, Chai B, Wang Q, et al (2013) FunGene: the functional gene pipeline and repository. Front Microbiol 4:. https://doi.org/10.3389/fmicb.2013.00291

Fu L, Niu B, Zhu Z, et al (2012) CD-HIT: accelerated for clustering the next-generation sequencing data. Bioinformatics 28:3150-3152. https://doi.org/10.1093/bioinformatics/bts565

Huang H, Jia Y, Sun G-X, Zhu Y-G (2012) Arsenic Speciation and Volatilization from Flooded Paddy Soils Amended with Different Organic Matters. Environ Sci Technol 46:2163-2168.

https://doi.org/10.1021/es203635s

Jackson BP (2015) Fast ion chromatography-ICP-QQQ for arsenic speciation. J Anal At Spectrom 30:1405-1407. https://doi.org/10.1039/C5JA00049A

Jia Y, Huang H, Sun G-X, et al (2012) Pathways and Relative Contributions to Arsenic Volatilization from Rice Plants and Paddy Soil. Environ Sci Technol 46:8090-8096. https://doi.org/10.1021/es300499a

Jia Y, Huang H, Zhong M, et al (2013) Microbial Arsenic Methylation in Soil and Rice Rhizosphere. Environ Sci Technol 47:3141-3148. https://doi.org/10.1021/es303649v

Kozlov AM, Darriba D, Flouri T, et al (2019) RAxML-NG: a fast, scalable and user-friendly tool for maximum likelihood phylogenetic inference. Bioinformatics 35:4453-4455.

https://doi.org/10.1093/bioinformatics/btz305

Kraska JE, Breitenbeck GA (2010) Simple, Robust Method for Quantifying Silicon in Plant Tissue. Communications in Soil Science and Plant Analysis 41:2075-2085.

https://doi.org/10.1080/00103624.2010.498537

Larsson A (2014) AliView: a fast and lightweight alignment viewer and editor for large datasets.

Bioinformatics 30:3276-3278. https://doi.org/10.1093/bioinformatics/btu531

Limmer MA, Mann J, Amaral DC, et al (2018) Silicon-rich amendments in rice paddies: Effects on arsenic uptake and biogeochemistry. Science of The Total Environment 624:1360-1368.

https://doi.org/10.1016/j.scitotenv.2017.12.207

Limmer MA, Seyfferth AL (2020) Carryover effects of silicon-rich amendments in rice paddies. Soil Sci Soc Am J saj2.20146. https://doi.org/10.1002/saj2.20146

Linam F, McCoach K, Limmer MA, Seyfferth AL (2021) Contrasting effects of rice husk pyrolysis temperature on silicon dissolution and retention of cadmium (Cd) and dimethylarsinic acid (DMA). Science of The Total Environment 765:144428

Liu W-J, McGrath SP, Zhao F-J (2014) Silicon has opposite effects on the accumulation of inorganic and methylated arsenic species in rice. Plant Soil 376:423-431. https://doi.org/10.1007/s11104-013-1991-7 
Lomax C, Liu W-J, Wu L, et al (2012) Methylated arsenic species in plants originate from soil microorganisms. New Phytologist 193:665-672. https://doi.org/10.1111/j.1469-8137.2011.03956.x

Lovley DR, Ueki T, Zhang T, et al (2011) Geobacter. In: Advances in Microbial Physiology. Elsevier, pp 1100

Luxton TP, Tadanier CJ, Eick MJ (2006) Mobilization of Arsenite by Competitive Interaction with Silicic Acid. Soil Sci Soc Am J 70:204-214. https://doi.org/10.2136/sssaj2005.0101

Ma R, Shen J, Wu J, et al (2014) Impact of agronomic practices on arsenic accumulation and speciation in rice grain. Environmental Pollution 194:217-223. https://doi.org/10.1016/j.envpol.2014.08.004

Maher W, Foster S, Krikowa F, et al (2013) Measurement of Inorganic Arsenic Species in Rice after Nitric Acid Extraction by HPLC-ICPMS: Verification Using XANES. Environ Sci Technol 47:5821-5827. https://doi.org/10.1021/es304299v

Marin AR, Masscheleyn PH, Patrick WH (1993) Soil redox-pH stability of arsenic species and its influence on arsenic uptake by rice. Plant Soil 152:245-253. https://doi.org/10.1007/BF00029094

McMurdie PJ, Holmes S (2013) phyloseq: An R Package for Reproducible Interactive Analysis and Graphics of Microbiome Census Data. PLoS ONE 8:e61217. https://doi.org/10.1371/journal.pone.0061217

Moore RM, Harrison AO, McAllister SM, et al (2017) Iroki: automatic customization and visualization of phylogenetic trees. Bioinformatics

Motuzova GV, Aptikaev RS, Karpova EA (2006) Fractionation of soil arsenic compounds. Eurasian Soil Sc 39:387-396. https://doi.org/10.1134/S1064229306040053

Oksanen J, Blanchet FG, Friendly M, et al (2019) vegan: Community Ecology Package

Park D, Kim H, Yoon S (2017) Nitrous Oxide Reduction by an Obligate Aerobic Bacterium, Gemmatimonas aurantiaca Strain T-27. Applied and Environmental Microbiology 83:12

Penido ES, Bennett AJ, Hanson TE, Seyfferth AL (2016) Biogeochemical impacts of silicon-rich rice residue incorporation into flooded soils: Implications for rice nutrition and cycling of arsenic. Plant Soil 13

Qin J, Rosen BP, Zhang Y, et al (2006) Arsenic detoxification and evolution of trimethylarsine gas by a microbial arsenite S-adenosylmethionine methyltransferase. Proc Natl Acad Sci U S A 103:2075-2080. https://doi.org/10.1073/pnas.0506836103

Quast C, Pruesse E, Yilmaz P, et al (2012) The SILVA ribosomal RNA gene database project: improved data processing and web-based tools. Nucleic Acids Research 41:D590-D596.

https://doi.org/10.1093/nar/gks1219

Page $17 / 30$ 
R Core Team (2019) R: A Language and Environment for Statistical Computing. R Foundation for Statistical Computing, Vienna, Austria

Roden EE, Wetzel RG (1996) Organic carbon oxidation and suppression of methane production by microbial Fe(III) oxide reduction in vegetated and unvegetated freshwater wetland sediments. Limnol Oceanogr 41:1733-1748. https://doi.org/10.4319/lo.1996.41.8.1733

Sanders JG (1979) Microbial role in the demethylation and oxidation of methylated arsenicals in seawater. Chemosphere 8:135-137. https://doi.org/10.1016/0045-6535(79)90061-4

Schloss PD, Westcott SL, Ryabin T, et al (2009) Introducing mothur: Open-Source, Platform-Independent, Community-Supported Software for Describing and Comparing Microbial Communities. Applied and Environmental Microbiology 75:7537-7541. https://doi.org/10.1128/AEM.01541-09

Seyfferth AL, Amaral D, Limmer MA, Guilherme LRG (2019a) Combined impacts of Si-rich rice residues and flooding extent on grain As and Cd in rice. Environment International 128:301-309.

https://doi.org/10.1016/j.envint.2019.04.060

Seyfferth AL, Fendorf S (2012) Silicate Mineral Impacts on the Uptake and Storage of Arsenic and Plant Nutrients in Rice (Oryza sativa L.). Environ Sci Technol 46:13176-13183.

https://doi.org/10.1021/es3025337

Seyfferth AL, Limmer M, Wu W (2019b) Si and Water Management Drives Changes in Fe and Mn Pools that Affect As Cycling and Uptake in Rice. Soil Syst 3:58. https://doi.org/10.3390/soilsystems3030058

Seyfferth AL, Limmer MA, Dykes GE (2018) On the Use of Silicon as an Agronomic Mitigation Strategy to Decrease Arsenic Uptake by Rice. In: Advances in Agronomy. Elsevier, pp 49-91

Seyfferth AL, Morris AH, Gill R, et al (2016) Soil Incorporation of Silica-Rich Rice Husk Decreases Inorganic Arsenic in Rice Grain. J Agric Food Chem 64:3760-3766.

https://doi.org/10.1021/acs.jafc.6b01201

Sowers TD, Adhikari D, Wang J, et al (2018a) Spatial Associations and Chemical Composition of Organic Carbon Sequestered in Fe, Ca, and Organic Carbon Ternary Systems. Environ Sci Technol 52:6936-6944. https://doi.org/10.1021/acs.est.8b01158

Sowers TD, Stuckey JW, Sparks DL (2018b) The synergistic effect of calcium on organic carbon sequestration to ferrihydrite. Geochem Trans 19:4. https://doi.org/10.1186/s12932-018-0049-4

Stookey LL (1970) Ferrozine--a new spectrophotometric reagent for iron. Anal Chem 42:779-781. https://doi.org/10.1021/ac60289a016

Takahashi Y, Minamikawa R, Hattori KH, et al (2004) Arsenic Behavior in Paddy Fields during the Cycle of Flooded and Non-flooded Periods. Environ Sci Technol 38:1038-1044. 
Taylor GJ, Crowder AA (1983) Use of the DCB Technique for Extraction of Hydrous Iron Oxides from Roots of Wetland Plants. American Journal of Botany 70:1254-1257. https://doi.org/10.2307/2443295

Teasley WA, Limmer MA, Seyfferth AL (2017) How Rice ( Oryza sativa L.) Responds to Elevated As under Different Si-Rich Soil Amendments. Environ Sci Technol 51:10335-10343.

https://doi.org/10.1021/acs.est.7b01740

Thomas F, Diaz-Bone RA, Wuerfel O, et al (2011) Connection between Multimetal(loid) Methylation in Methanoarchaea and Central Intermediates of Methanogenesis囚. Appl Environ Microbiol 77:8669-8675. https://doi.org/10.1128/AEM.06406-11

Tremblay J, Singh K, Fern A, et al (2015) Primer and platform effects on 16S rRNA tag sequencing. Front Microbiol 6:. https://doi.org/10.3389/fmicb.2015.00771

Viacava K, Meibom KL, Ortega D, et al (2020) Variability in Arsenic Methylation Efficiency across Aerobic and Anaerobic Microorganisms. Environ Sci Technol 9

Von Endt DW, Kearney PC, Kaufman DD (1968) Degradation of MSMA by soil microorganisms. J Agric Food Chem 16:17-20. https://doi.org/10.1021/jf60155a017

Wang M, Tang Z, Chen X-P, et al (2019) Water management impacts the soil microbial communities and total arsenic and methylated arsenicals in rice grains. Environmental Pollution 247:736-744. https://doi.org/10.1016/j.envpol.2019.01.043

Weiss JV, Rentz JA, Plaia T, et al (2007) Characterization of Neutrophilic Fe(II)-Oxidizing Bacteria Isolated from the Rhizosphere of Wetland Plants and Description of Ferritrophicum radicicola gen. nov. sp. nov., and Sideroxydans paludicola sp. nov. Geomicrobiology Journal 24:559-570.

https://doi.org/10.1080/01490450701670152

Yan Y, Ye J, Xue X-M, Zhu Y-G (2015) Arsenic Demethylation by a C.As Lyase in Cyanobacterium Nostoc sp. PCC 7120. Environ Sci Technol 49:14350-14358. https://doi.org/10.1021/acs.est.5b03357

Yang Y-P, Zhang H-M, Yuan H-Y, et al (2018) Microbe mediated arsenic release from iron minerals and arsenic methylation in rhizosphere controls arsenic fate in soil-rice system after straw incorporation. Environmental Pollution 236:598-608. https://doi.org/10.1016/j.envpol.2018.01.099

Zeng Y, Selyanin V, Lukeš M, et al (2015) Characterization of the microaerophilic, bacteriochlorophyll acontaining bacterium Gemmatimonas phototrophica sp. nov., and emended descriptions of the genus Gemmatimonas and Gemmatimonas aurantiaca. International Journal of Systematic and Evolutionary Microbiology 65:2410-2419. https://doi.org/10.1099/ijs.0.000272 
Zhang H, Sekiguchi Y, Hanada S, et al Gemmatimonas aurantiaca gen. nov., sp. nov., a Gram-negative, aerobic, polyphosphate- accumulating micro-organism, the first cultured representative of the new bacterial phylum Gemmatimonadetes phyl. nov. International Journal of Systematic and Evolutionary Microbiology 9

Zhang S-Y, Zhao F-J, Sun G-X, et al (2015) Diversity and Abundance of Arsenic Biotransformation Genes in Paddy Soils from Southern China. Environ Sci Technol 49:4138-4146.

https://doi.org/10.1021/acs.est.5b00028

Zhao F-J, Harris E, Yan J, et al (2013a) Arsenic Methylation in Soils and Its Relationship with Microbial arsM Abundance and Diversity, and As Speciation in Rice. Environ Sci Technol 47:7147-7154. https://doi.org/10.1021/es304977m

Zhao F-J, Zhu Y-G, Meharg AA (2013b) Methylated Arsenic Species in Rice: Geographical Variation, Origin, and Uptake Mechanisms. Environ Sci Technol 47:3957-3966. https://doi.org/10.1021/es304295n

(2016) Database resources of the National Center for Biotechnology Information. Nucleic Acids Res 44:D7-D19. https://doi.org/10.1093/nar/gkv1290

\section{Figures}
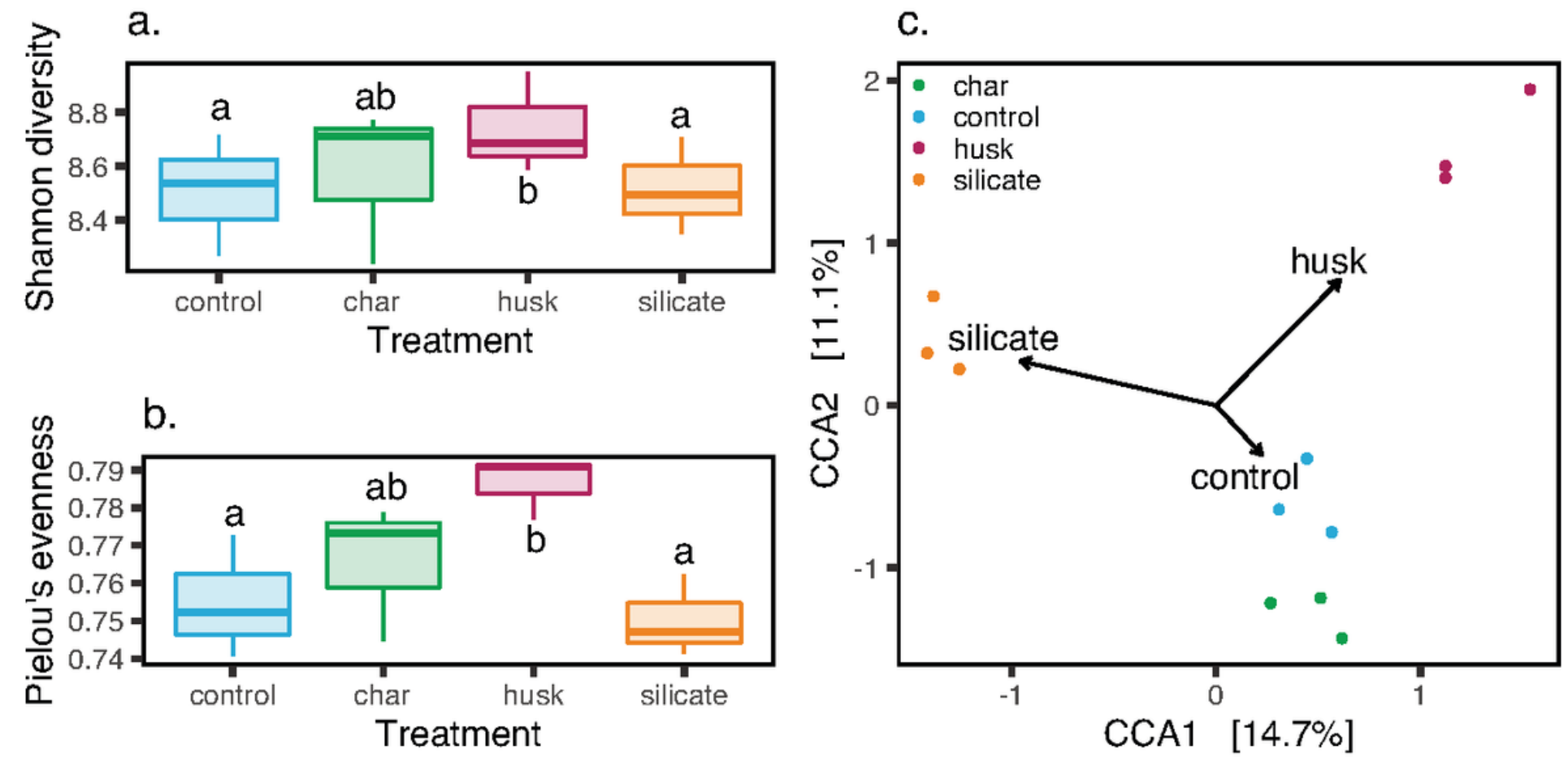

Figure 1

Alpha diversity of $16 \mathrm{~S}$ communities by treatment $(\mathrm{A})$ and constrained correspondence analysis of $16 \mathrm{~S}$ communities at ripening (88 DPT) in the rhizosphere (B) from rice paddies amended with various Si-rich 


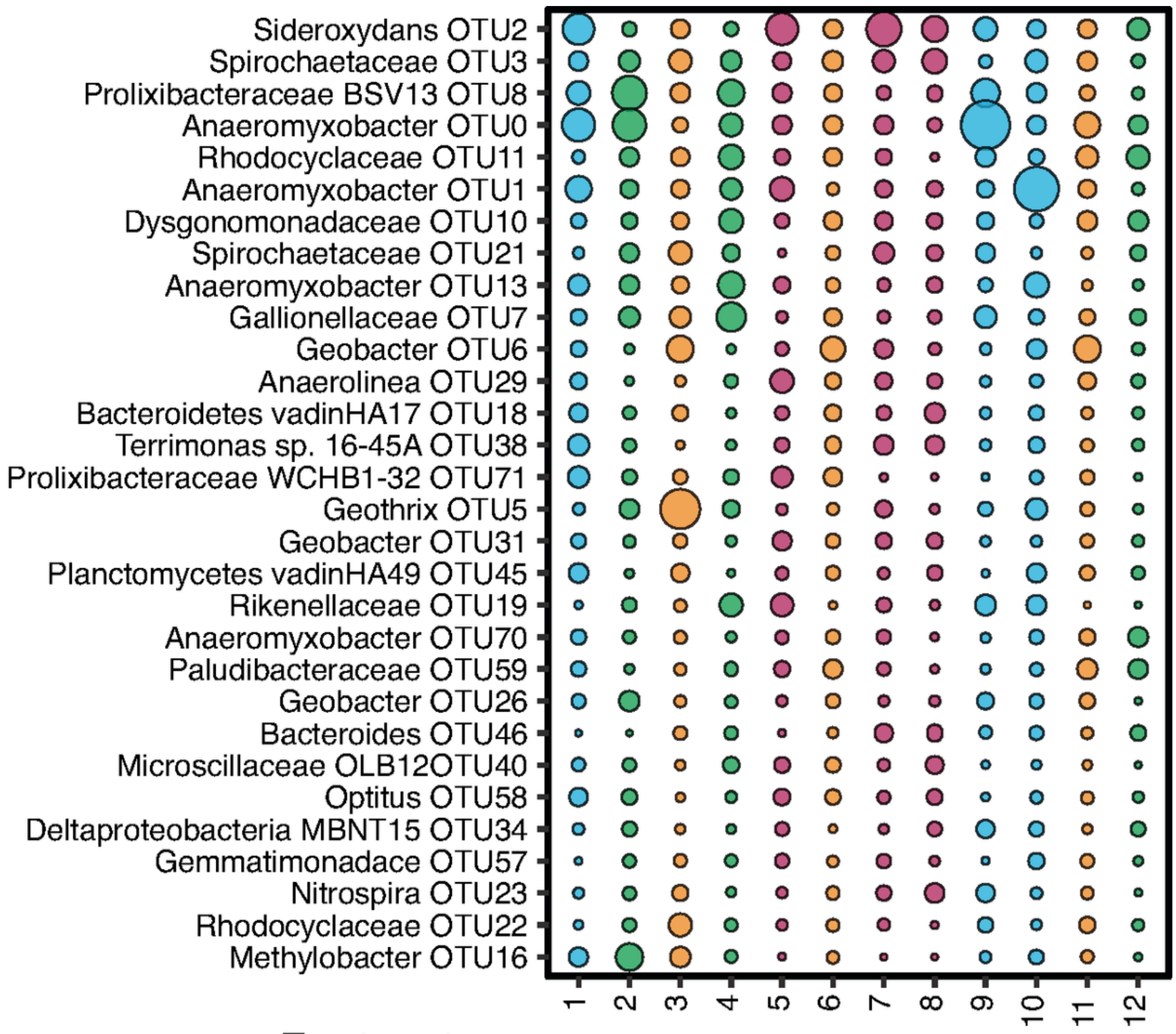

Treatment $\bigcirc$ control $\bigcirc$ husk $\bigcirc$ char $\bigcirc$ silicate

Relative Abundance $\bigcirc 10.0 \% \circ 1.0 \%$ ○ $0.1 \%$

Figure 2

Bubble plot of the thirty most abundant 16S OTUs in the rhizosphere at grain ripening. Size of bubble corresponds to relative abundance, with bubbles colored according to treatment. X-axis denotes paddy number. 


\section{paddy}

\begin{tabular}{|c|c|c|c|c|c|c|c|c|c|c|c|c|c|}
\hline ny & & 12 & 2 & 4 & 1 & 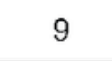 & & & & 8 & 11 & 3 & 6 \\
\hline ets & 7 & & $\%$ & $\%$ & $\%$ & 0 & c & $\%$ & $\%$ & $\%$ & & $\%$ & 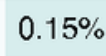 \\
\hline Holop & 92 & $\%$ & $08 \%$ & 13\% & $03 \%$ & $0.15 \%$ & $0.06 \%$ & $0.06 \%$ & $0.06 \%$ & $0.02 \%$ & & $2 \%$ & $03^{\circ}$ \\
\hline$S p$ & 07 & & $\%$ & $\%$ & $\%$ & $\%$ & 0 & & & & & & \\
\hline Palu & 72 & $\%$ & $0.07 \%$ & $0.05 \%$ & $0.16 \%$ & $0.17 \%$ & $0.06 \%$ & $0.02 \%$ & $0.05 \%$ & $0.03 \%$ & $\%$ & $.06 \%$ & $04^{\circ}$ \\
\hline Meth & 86 & $\%$ & $26 \%$ & $0.18 \%$ & $0.19 \%$ & $0.11 \%$ & $7 \%$ & $0.25 \%$ & $0.31 \%$ & $0.62 \%$ & & $3 \%$ & $6 \%$ \\
\hline ent & 92 & $\%$ & $0.13 \%$ & $10 \%$ & $0.17 \%$ & 0 & $0.05 \%$ & $\%$ & $\%$ & $\%$ & $\%$ & $\%$ & $0.16 \%$ \\
\hline A & 37 & $\%$ & $0.03 \%$ & $13 \%$ & $0.28 \%$ & $0.00 \%$ & $0.12 \%$ & $0.53 \%$ & $0.39 \%$ & $1.12 \%$ & $\%$ & $\%$ & $0.10 \%$ \\
\hline Korik & 32 & & $0.34 \%$ & $0.17 \%$ & $0.10 \%$ & $0.00 \%$ & $0.02 \%$ & $\%$ & $\%$ & $\%$ & $\%$ & $\%$ & $0.05 \%$ \\
\hline Lent & 89 & $\%$ & $4 \%$ & $2 \%$ & $23 \%$ & $2 \%$ & $0.06 \%$ & $\%$ & $\%$ & $\%$ & & $\%$ & 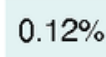 \\
\hline $\mathrm{Geo}$ & 6 & & $0.13 \%$ & & $0.64 \%$ & 0 & $\%$ & & & $\%$ & & & \\
\hline$R$ & 22 & & $\%$ & $3 \%$ & $\%$ & $\%$ & 0 & $\%$ & $\%$ & $\%$ & & $\%$ & $0.33 \%$ \\
\hline Lacu & 41 & & $0.16 \%$ & 0. & 0 & 0. & 0 & 0 & $\%$ & $\%$ & $\%$ & & 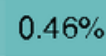 \\
\hline Fibrc & 27 & $\%$ & $23 \%$ & $\%$ & $12 \%$ & 0 & $98 \%$ & 0 & $\%$ & $0.12 \%$ & 1. & $\%$ & 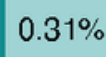 \\
\hline A & 79 & 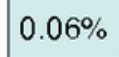 & c & ( & $\%$ & 0 & 0 & 0 & $\%$ & & & $\%$ & $021^{\circ}$ \\
\hline roscil & 06 & & $0.10 \%$ & $0.17 \%$ & $0.03 \%$ & $0.36 \%$ & $0.08 \%$ & $0.02 \%$ & $0.03 \%$ & $0.00 \%$ & $\%$ & & $\%$ \\
\hline Porphyrobacte & 22 & & c & $c$ & 0 & 0 & 0 & 0 & $\%$ & & & & \\
\hline nmat & 170 & $5 \%$ & $11 \%$ & $10 \%$ & $0.03 \%$ & 0. & $0.02 \%$ & 0. & $0.08 \%$ & $\%$ & & & ( \\
\hline Paludibacterace & 8 & & c & ( & & 0 & 0 & & & & & & \\
\hline oku & 187 & $6 \%$ & $18 \%$ & $6 \%$ & $0.07 \%$ & $0.07 \%$ & $0.09 \%$ & 0. & $\%$ & 0.0 & $\%$ & $\%$ & 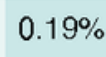 \\
\hline$-\pi$ & $1-$ & 6 & 0 & 0 & 0 & 0 & 0 & & & & & & \\
\hline 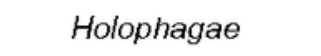 & 5 & , & $\%$ & $c$ & ( & 0 & 0 & 0 & $\%$ & $\%$ & $\%$ & 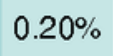 & $0.12 \%$ \\
\hline riok & 427 & $\%$ & $0.01 \%$ & 0. & 0 & 0 & 0 & & & & & 0. & 0 \\
\hline Ery & 55 & 6 & 6 & c & ( & 0 & 0 & 0 & 0 & $0.00 \%$ & & $\%$ & 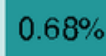 \\
\hline G & 66 & $\%$ & $0.05 \%$ & 0.0 & 0. & 0 & 0.0 & & & & & & \\
\hline yed & 286 & $\%$ & $\%$ & $\%$ & $\%$ & $\%$ & c & & & $\%$ & $\%$ & $\%$ & \\
\hline
\end{tabular}

paddy treatment

${ }_{10^{\text {th }}}^{90^{\text {th }} \text { percentile }} \square$ char $\square$ control $\square$ husk $\square$ silicate

\section{Figure 3}

Relative abundance heatmap of $16 \mathrm{~S}$ indicator species for each paddy with median relative abundance greater that $0.05 \%$ in rhizosphere at grain ripening (88 DPT). The treatment of each paddy is color-coded underneath the chart, and the treatment the OTU was an indicator of is color-coded to the right of the chart. The heatmap is colored according to relative abundance, with relative abundances in the 90th percentile in teal, and abundances in the 10th percentile or lower in white. 


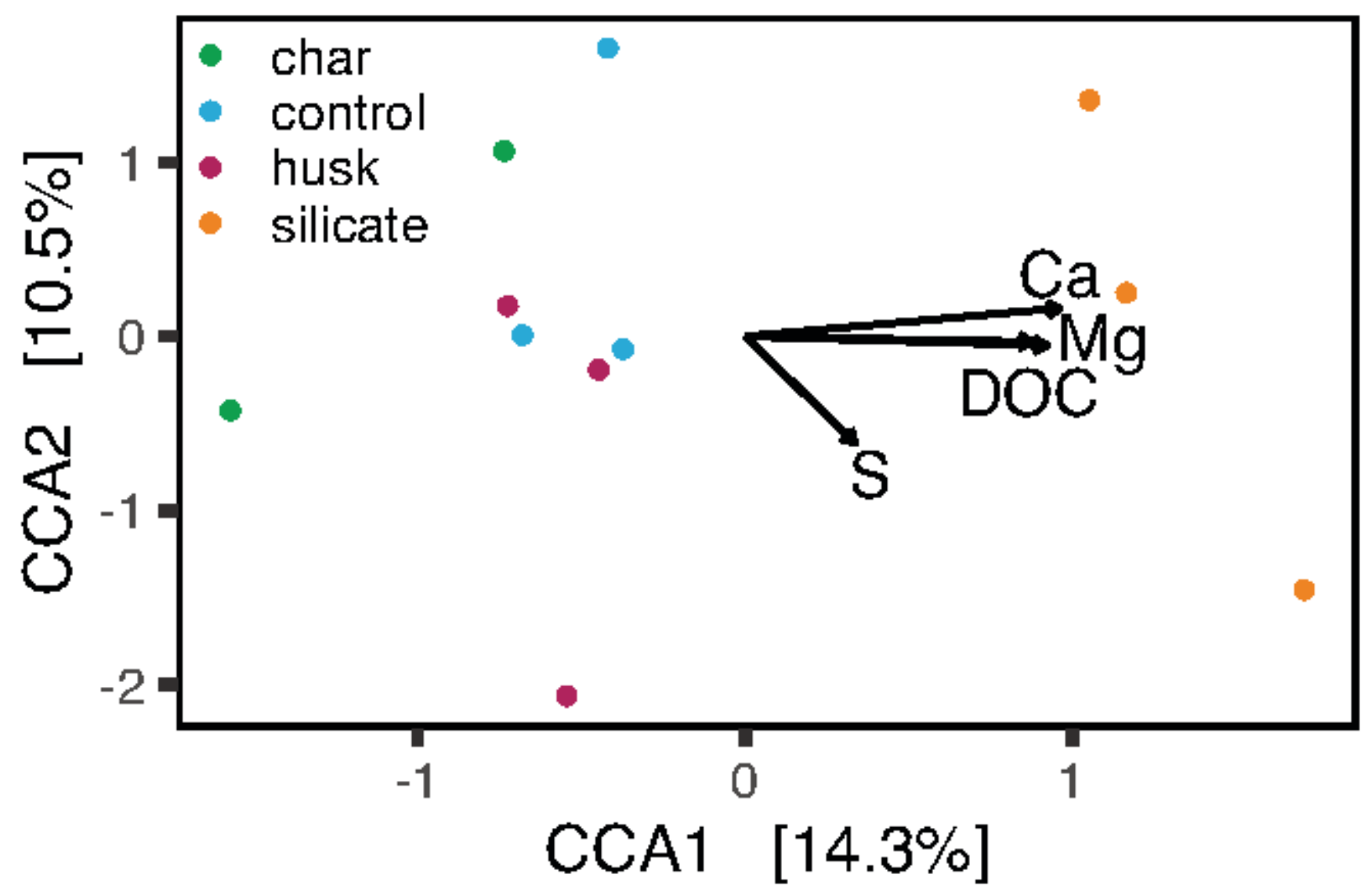

Figure 4

Constrained correspondence analysis with porewater variables that significantly correlated with differences in $16 \mathrm{~S}$ microbial community composition at rhizosphere ripening. Samples are represented as points in CCA and constraining variables are overlain as arrows.

a.

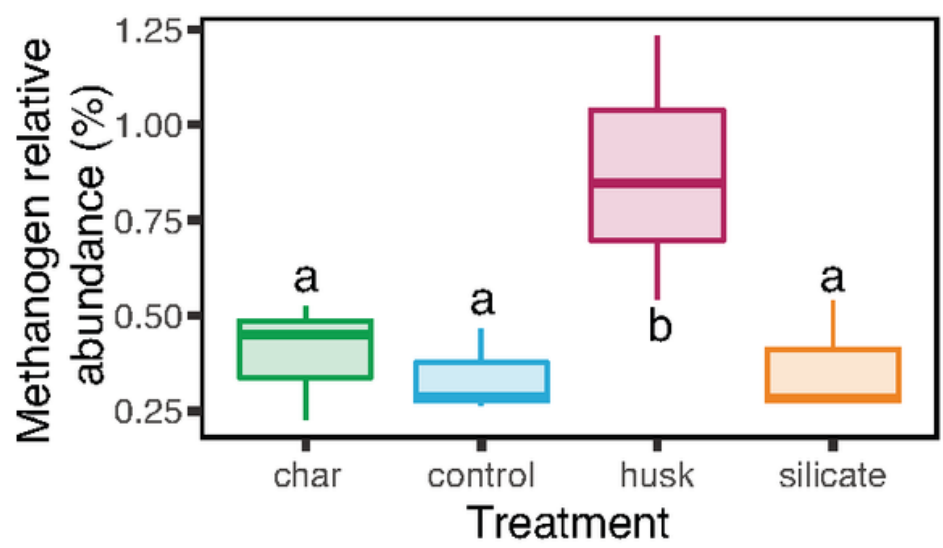

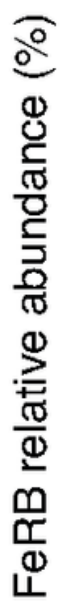

b.

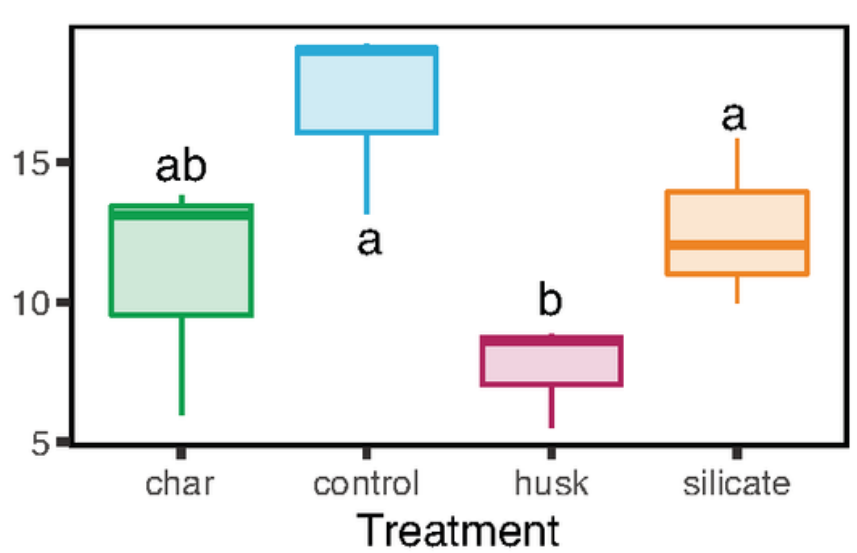

Figure 5

Boxplots showing summed relative abundance of methanogens (A) and FeRB (B). In A and B different letters indicate groups that are significantly different from one another. 

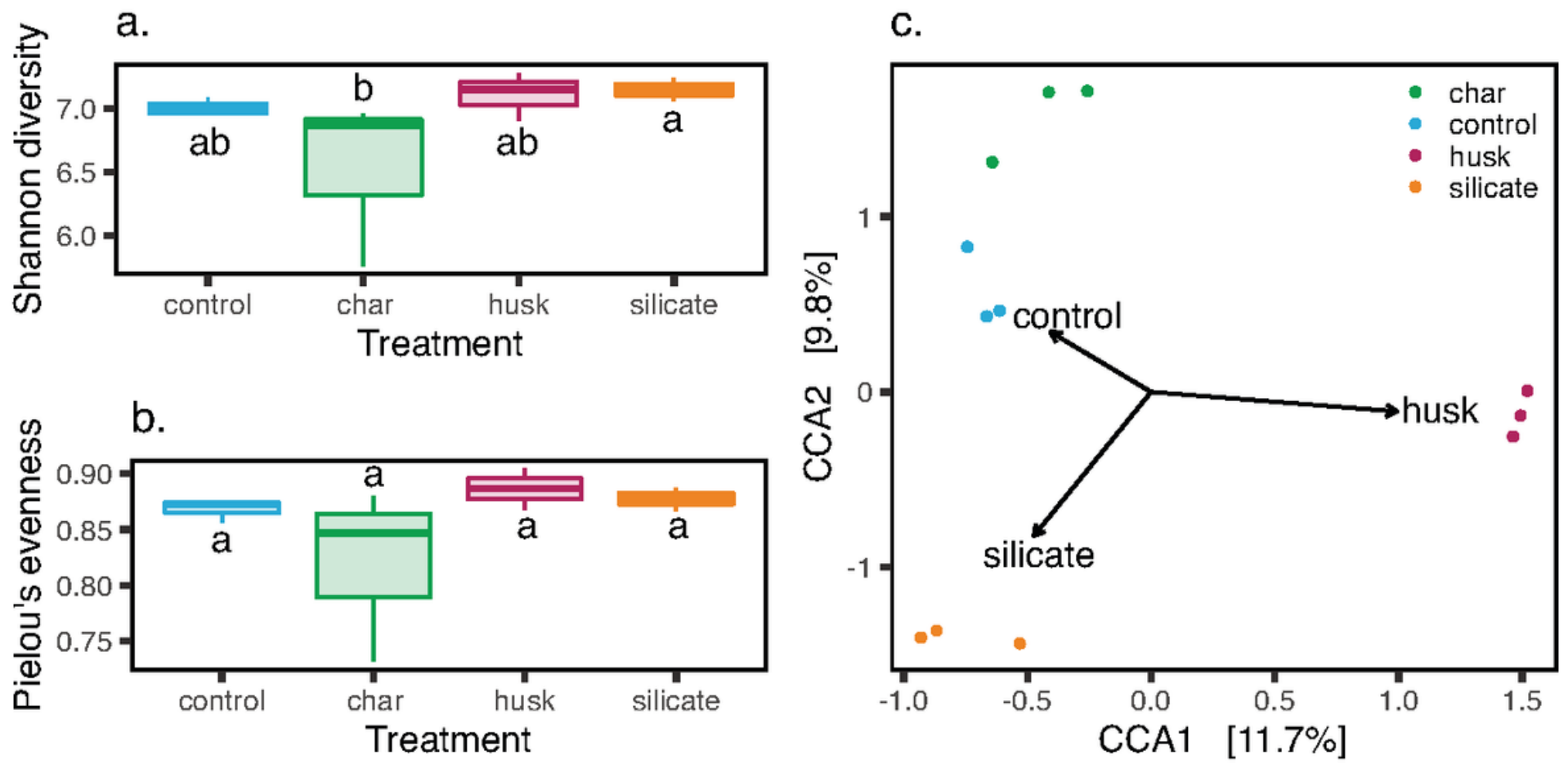

Figure 6

Alpha diversity boxplots of arsM by treatment $(\mathrm{A})$ and constrained correspondence analysis of arsM communities at ripening (88 DPT) in the rhizosphere (B) from rice paddies amended with various Si-rich sources and unamended controls. In alpha diversity plots data with the same letters above the bars are not significantly different from one another. Samples are represented as points in CCA where treatment constrained the ordination, with treatment arrows overlain. 


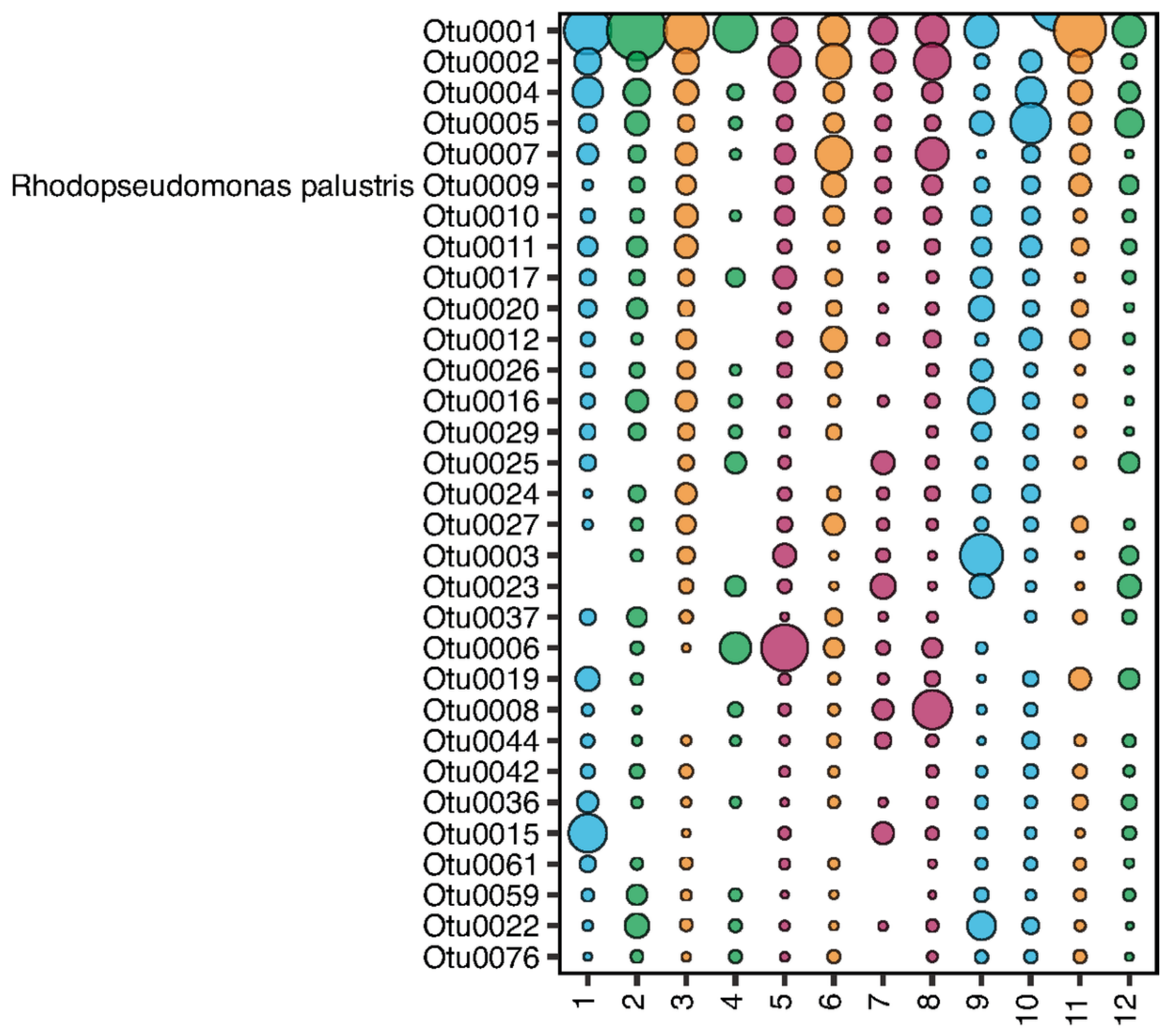

Treatment $\bigcirc$ control $\bigcirc$ husk $\bigcirc$ char $\bigcirc$ silicate Relative Abundance $\bigcirc 10.0 \% \circ 1.0 \% \circ 0.1 \%$

Figure 7

Bubble chart of the most abundant arsM sequences in the rhizosphere at grain ripening. Size of bubble corresponds to relative abundance, with bubbles colored according to treatment. 
paddy

\begin{tabular}{|c|c|c|c|c|c|c|c|c|c|c|c|}
\hline OTU & 2 & 4 & 1 & 9 & 10 & 5 & 7 & 8 & 11 & 3 & 6 \\
\hline $0.00 \%$ & $1.13 \%$ & $2.06 \%$ & $0.00 \%$ & $0.00 \%$ & $0.13 \%$ & $0.07 \%$ & $0.00 \%$ & $0.00 \%$ & $0.14 \%$ & $0.00 \%$ & $0.07 \%$ \\
\hline $0.06 \%$ & $0.00 \%$ & $0.00 \%$ & $0.11 \%$ & $0.16 \%$ & $0.00 \%$ & $0.00 \%$ & $0.00 \%$ & $0.00 \%$ & $0.00 \%$ & $0.00 \%$ & $0.00 \%$ \\
\hline $0.12 \%$ & $0.00 \%$ & $0.00 \%$ & $0.11 \%$ & $0.05 \%$ & $0.00 \%$ & $0.03 \%$ & $0.00 \%$ & $0.00 \%$ & $0.00 \%$ & $0.00 \%$ & $0.00 \%$ \\
\hline $0.06 \%$ & $0.00 \%$ & $0.00 \%$ & $0.06 \%$ & $0.05 \%$ & $0.00 \%$ & $0.00 \%$ & $0.00 \%$ & $0.00 \%$ & $0.00 \%$ & $0.00 \%$ & $0.00 \%$ \\
\hline $0.00 \%$ & $0.07 \%$ & $0.51 \%$ & $0.28 \%$ & $0.11 \%$ & $0.39 \%$ & $0.30 \%$ & $1.46 \%$ & $7.02 \%$ & $0.00 \%$ & $0.00 \%$ & $0.27 \%$ \\
\hline $0.23 \%$ & $0.07 \%$ & $0.17 \%$ & $0.33 \%$ & $0.00 \%$ & $0.13 \%$ & $0.36 \%$ & $0.83 \%$ & $0.27 \%$ & $0.00 \%$ & $0.00 \%$ & $0.00 \%$ \\
\hline $0.17 \%$ & $0.00 \%$ & $0.00 \%$ & $0.17 \%$ & $0.16 \%$ & $0.04 \%$ & $0.33 \%$ & $1.25 \%$ & $0.64 \%$ & $0.00 \%$ & $0.00 \%$ & $0.00 \%$ \\
\hline $0.23 \%$ & $0.07 \%$ & $0.00 \%$ & $0.39 \%$ & $0.00 \%$ & $0.09 \%$ & $1.56 \%$ & $1.14 \%$ & $0.10 \%$ & $0.05 \%$ & $0.00 \%$ & $0.07 \%$ \\
\hline $0.17 \%$ & $0.00 \%$ & $0.00 \%$ & $0.00 \%$ & $0.11 \%$ & $0.04 \%$ & $0.10 \%$ & $2.39 \%$ & $0.14 \%$ & $0.14 \%$ & $0.00 \%$ & $0.00 \%$ \\
\hline $0.06 \%$ & $0.14 \%$ & $0.17 \%$ & $0.06 \%$ & $0.00 \%$ & $0.00 \%$ & $0.13 \%$ & $0.73 \%$ & $0.14 \%$ & $0.00 \%$ & $0.05 \%$ & $0.00 \%$ \\
\hline $0.06 \%$ & $0.00 \%$ & $0.17 \%$ & $0.00 \%$ & $0.05 \%$ & $0.00 \%$ & $0.56 \%$ & $1.98 \%$ & $0.10 \%$ & $0.00 \%$ & $0.00 \%$ & $0.00 \%$ \\
\hline $0.06 \%$ & $0.00 \%$ & $0.00 \%$ & $0.06 \%$ & $0.00 \%$ & $0.04 \%$ & $0.20 \%$ & $0.31 \%$ & $0.14 \%$ & $0.00 \%$ & $0.00 \%$ & $0.00 \%$ \\
\hline $0.00 \%$ & $0.00 \%$ & $0.51 \%$ & $0.00 \%$ & $0.00 \%$ & $0.00 \%$ & $2.12 \%$ & $0.42 \%$ & $0.41 \%$ & $0.00 \%$ & $0.05 \%$ & $0.00 \%$ \\
\hline $0.00 \%$ & $0.00 \%$ & $0.00 \%$ & $0.06 \%$ & $0.00 \%$ & $0.00 \%$ & $0.07 \%$ & $2.50 \%$ & $0.14 \%$ & $0.00 \%$ & $0.00 \%$ & $0.07 \%$ \\
\hline $0.00 \%$ & $0.00 \%$ & $0.00 \%$ & $0.11 \%$ & $0.00 \%$ & $0.00 \%$ & $0.56 \%$ & $0.42 \%$ & $0.07 \%$ & $0.00 \%$ & $0.00 \%$ & $0.07 \%$ \\
\hline $0.12 \%$ & $0.00 \%$ & $0.00 \%$ & $0.00 \%$ & $0.00 \%$ & $0.00 \%$ & $0.03 \%$ & $1.14 \%$ & $0.14 \%$ & $0.09 \%$ & $0.00 \%$ & $0.00 \%$ \\
\hline $0.00 \%$ & $0.00 \%$ & $0.00 \%$ & $0.00 \%$ & $0.00 \%$ & $0.00 \%$ & $0.17 \%$ & $0.31 \%$ & $0.37 \%$ & $0.00 \%$ & $0.00 \%$ & $0.00 \%$ \\
\hline $0.00 \%$ & $0.00 \%$ & $0.00 \%$ & $0.00 \%$ & $0.05 \%$ & $0.00 \%$ & $0.53 \%$ & $0.10 \%$ & $0.03 \%$ & $0.00 \%$ & $0.00 \%$ & $0.00 \%$ \\
\hline $0.00 \%$ & $0.00 \%$ & $0.00 \%$ & $0.00 \%$ & $0.00 \%$ & $0.09 \%$ & $0.26 \%$ & $0.10 \%$ & $0.20 \%$ & $0.00 \%$ & $0.00 \%$ & $0.00 \%$ \\
\hline $0.00 \%$ & $0.00 \%$ & $0.17 \%$ & $0.00 \%$ & $0.00 \%$ & $0.00 \%$ & $0.23 \%$ & $0.21 \%$ & $0.17 \%$ & $0.09 \%$ & $0.00 \%$ & $0.00 \%$ \\
\hline $0.00 \%$ & $0.00 \%$ & $0.00 \%$ & $0.00 \%$ & $0.00 \%$ & $0.00 \%$ & $0.10 \%$ & $0.73 \%$ & $0.03 \%$ & $0.00 \%$ & $0.00 \%$ & $0.00 \%$ \\
\hline $0.00 \%$ & $0.00 \%$ & $0.00 \%$ & $0.00 \%$ & $0.00 \%$ & $0.04 \%$ & $0.07 \%$ & $0.10 \%$ & $0.20 \%$ & $0.00 \%$ & $0.00 \%$ & $0.00 \%$ \\
\hline $0.00 \%$ & $0.00 \%$ & $0.00 \%$ & $0.00 \%$ & $0.00 \%$ & $0.00 \%$ & $0.07 \%$ & $0.31 \%$ & $0.03 \%$ & $0.05 \%$ & $0.00 \%$ & $0.00 \%$ \\
\hline $0.00 \%$ & $0.00 \%$ & $0.00 \%$ & $0.00 \%$ & $0.00 \%$ & $0.00 \%$ & $0.07 \%$ & $0.21 \%$ & $0.07 \%$ & $0.00 \%$ & $0.00 \%$ & $0.00 \%$ \\
\hline $0.00 \%$ & $0.00 \%$ & $0.17 \%$ & $0.00 \%$ & $0.00 \%$ & $0.00 \%$ & $0.07 \%$ & $0.21 \%$ & $0.03 \%$ & $0.00 \%$ & $0.00 \%$ & $0.00 \%$ \\
\hline $0.00 \%$ & $0.00 \%$ & $0.00 \%$ & $0.00 \%$ & $0.00 \%$ & $0.00 \%$ & $0.03 \%$ & $0.10 \%$ & $0.07 \%$ & $0.00 \%$ & $0.00 \%$ & $0.00 \%$ \\
\hline $0.00 \%$ & $0.00 \%$ & $0.00 \%$ & $0.00 \%$ & $0.00 \%$ & $0.00 \%$ & $0.07 \%$ & $0.10 \%$ & $0.03 \%$ & $0.00 \%$ & $0.00 \%$ & $0.00 \%$ \\
\hline $0.00 \%$ & $0.00 \%$ & $0.00 \%$ & $0.00 \%$ & $0.00 \%$ & $0.00 \%$ & $0.03 \%$ & $0.10 \%$ & $0.03 \%$ & $0.00 \%$ & $0.00 \%$ & $0.00 \%$ \\
\hline $0.23 \%$ & $0.21 \%$ & $0.00 \%$ & $0.44 \%$ & $0.37 \%$ & $1.74 \%$ & $0.60 \%$ & $0.31 \%$ & $0.81 \%$ & $1.17 \%$ & $1.20 \%$ & $2.46 \%$ \\
\hline $0.06 \%$ & $0.00 \%$ & $0.00 \%$ & $0.28 \%$ & $0.16 \%$ & $0.48 \%$ & $0.10 \%$ & $0.31 \%$ & $0.24 \%$ & $0.09 \%$ & $0.32 \%$ & $1.30 \%$ \\
\hline $0.12 \%$ & $0.14 \%$ & $0.00 \%$ & $0.11 \%$ & $0.11 \%$ & $0.74 \%$ & $0.13 \%$ & $0.00 \%$ & $0.20 \%$ & $0.23 \%$ & $0.74 \%$ & $0.48 \%$ \\
\hline $0.00 \%$ & $0.00 \%$ & $0.17 \%$ & $0.06 \%$ & $0.00 \%$ & $0.22 \%$ & $0.00 \%$ & $0.00 \%$ & $0.03 \%$ & $0.14 \%$ & $0.18 \%$ & $0.14 \%$ \\
\hline $0.00 \%$ & $0.00 \%$ & $0.00 \%$ & $0.06 \%$ & $0.05 \%$ & $0.09 \%$ & $0.00 \%$ & $0.00 \%$ & $0.03 \%$ & $0.00 \%$ & $0.14 \%$ & $0.14 \%$ \\
\hline $0.00 \%$ & $0.00 \%$ & $0.00 \%$ & $0.00 \%$ & $0.00 \%$ & $0.04 \%$ & $0.00 \%$ & $0.00 \%$ & $0.00 \%$ & $0.00 \%$ & $0.05 \%$ & $0.07 \%$ \\
\hline
\end{tabular}

\begin{tabular}{|l}
$90^{\text {th }}$ percentile \\
${ }_{10^{\text {th }}}$ percentile
\end{tabular} char $\square$ control $\square$ husk $\square$ silicate

\section{Figure 8}

Relative abundance heatmap of arsM indicators species for each paddy in rhizosphere at grain ripening (88 DPT). The treatment of each paddy is color-coded underneath the chart, and the treatment the OTU was an indicator of is color-coded to the right of the chart. The heatmap is colored according to relative abundance, with relative abundances in the 90th percentile in teal, and abundances in the 10th percentile or lower in white. 


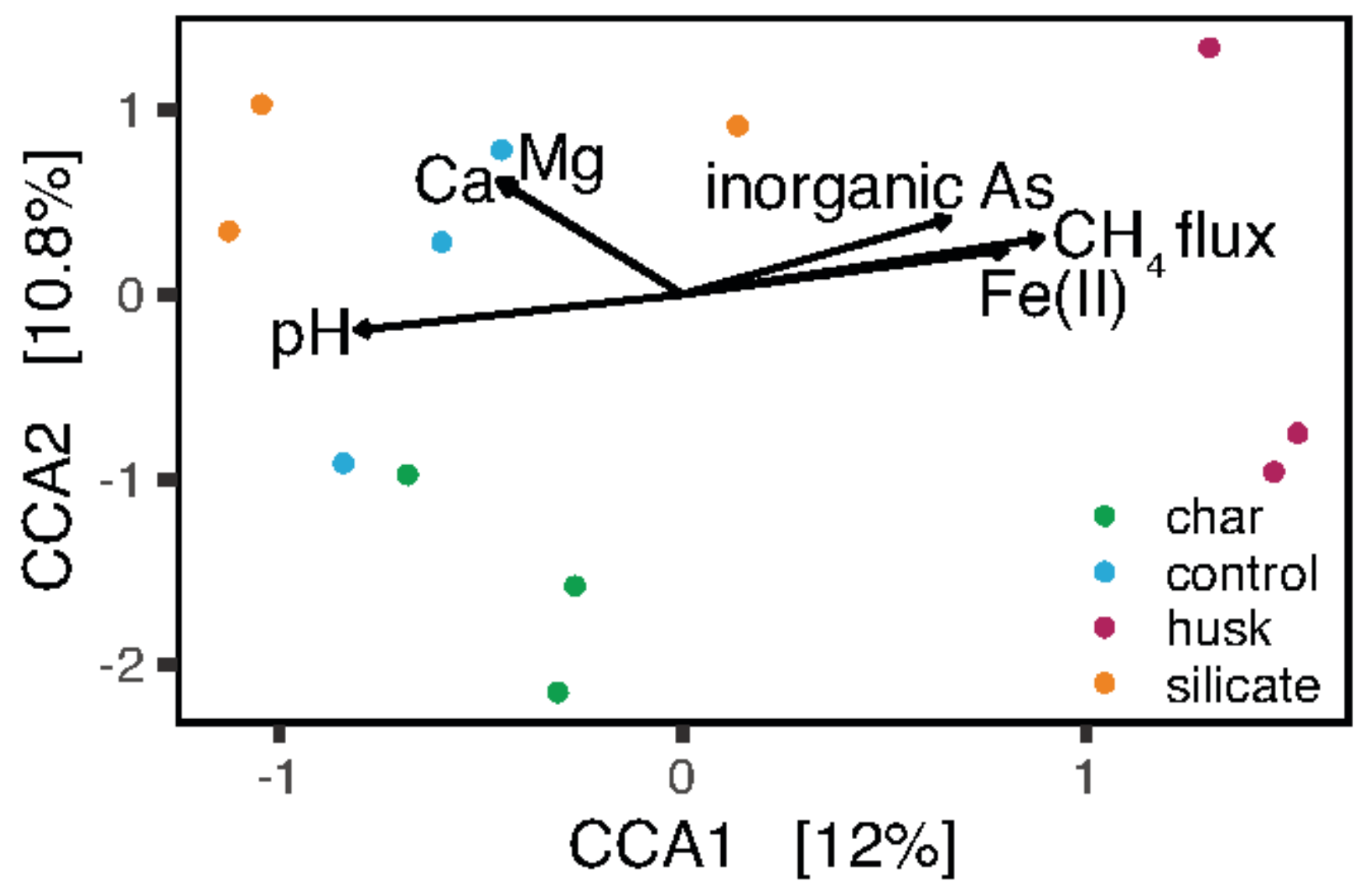

Figure 9

Constrained correspondence analysis with porewater $\mathrm{Ca}(\mu \mathrm{M})$, methane flux (nmol/m2/s), $\mathrm{Mg}(\mu \mathrm{M}), \mathrm{pH}$, inorganic As $(\mu \mathrm{M})$, and Fe(II) $(\mu \mathrm{M})$, correlating with differences in arsM community composition. Samples are represented as points in CCA and constraining variables are overlain as arrows. 
a.

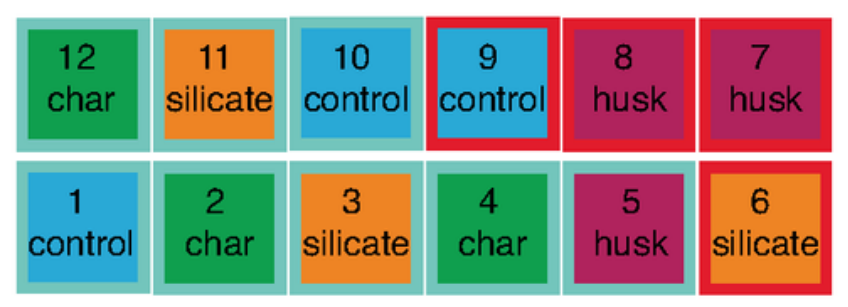

c. $\operatorname{arsM}$
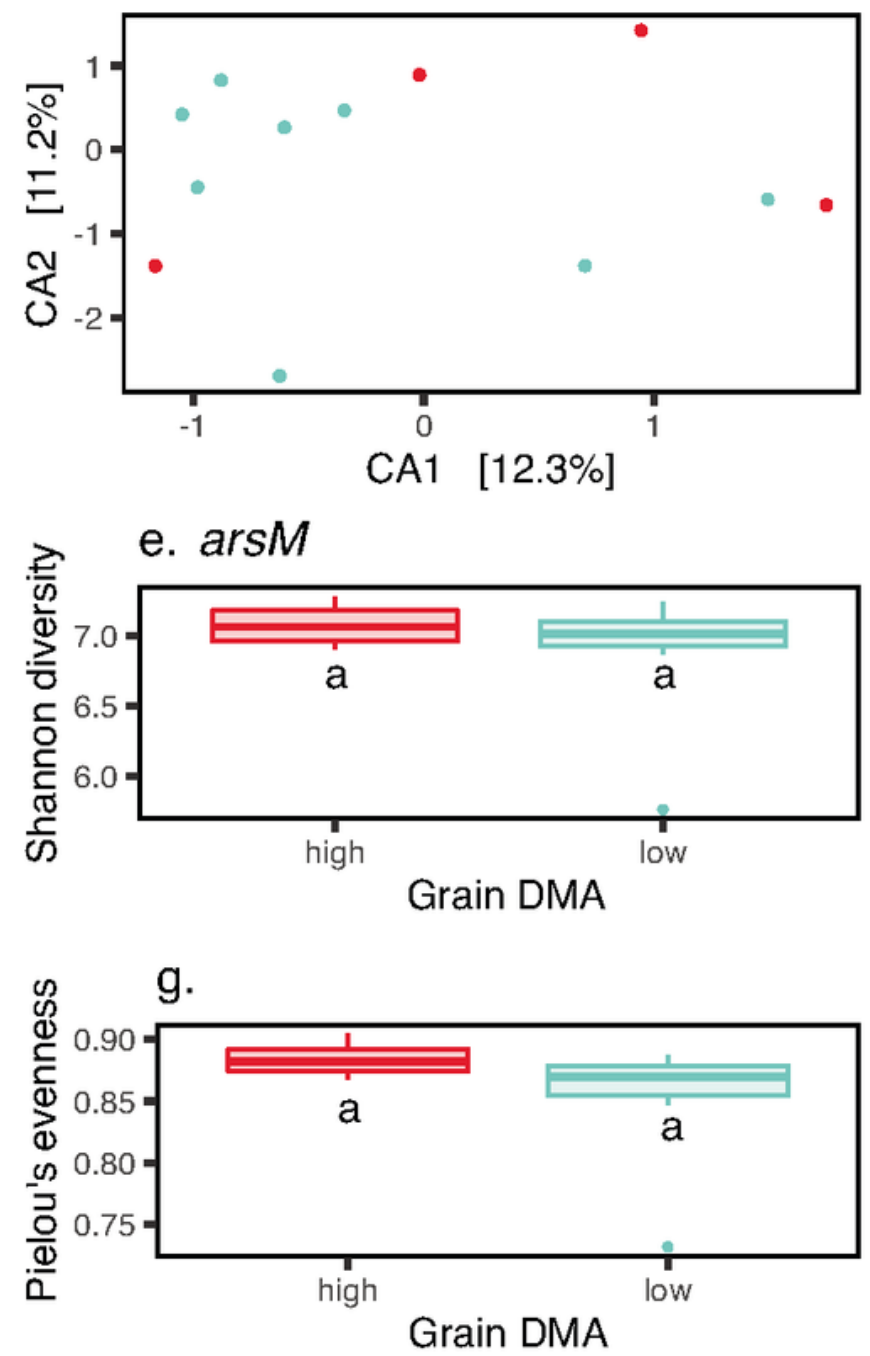

b.

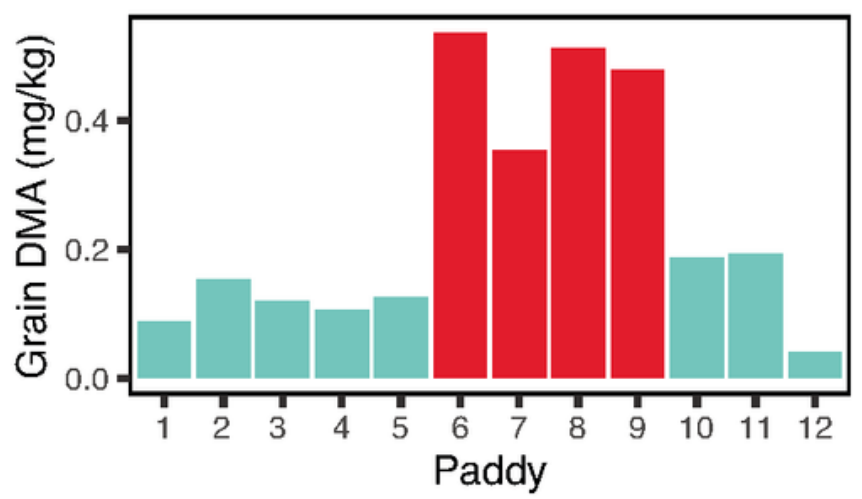

d. $16 \mathrm{~S}$
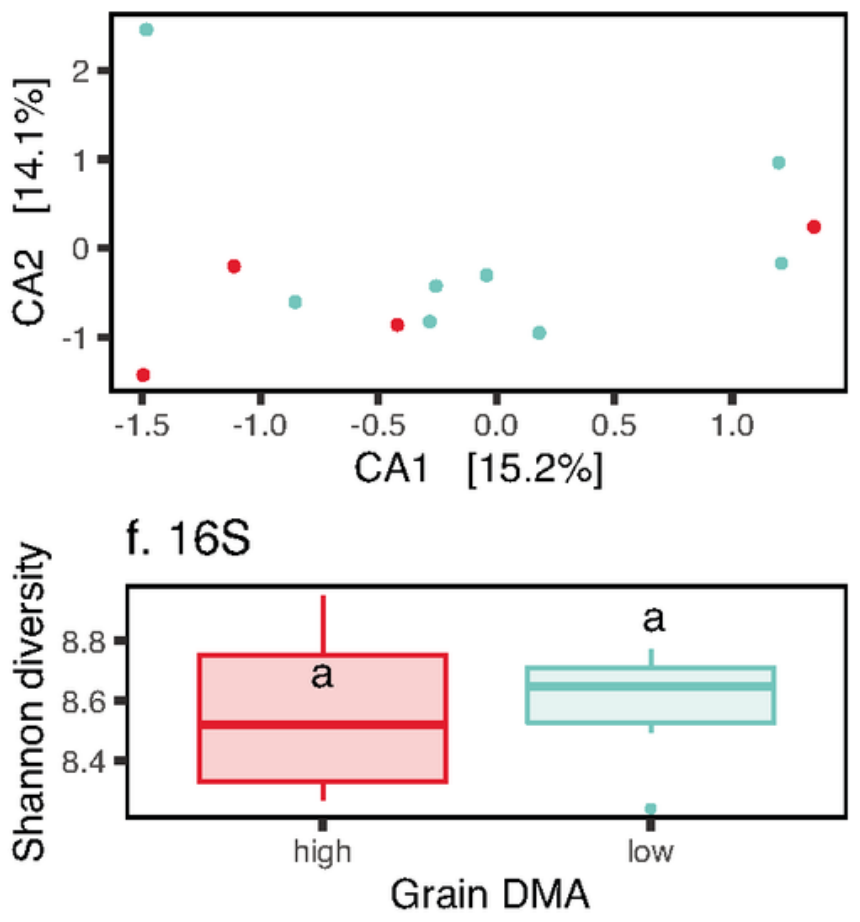

h.

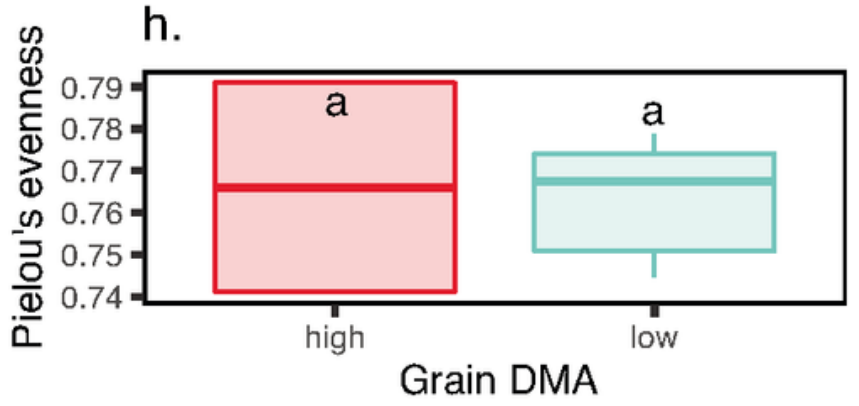

\section{Figure 10}

Grain DMA varied more by spatial orientation of paddy location than by treatment. A. Diagram of paddy field layout. B. Grain DMA by paddy, colored to represent assigned grain As category. C, D. Correspondence analysis of arsM (C) and 16S (D) microbial community composition colored by grain DMA category. E, F. Boxplot comparing alpha diversity compared across grain DMA categories, with 
outliers as points for arsM $(\mathrm{E}, \mathrm{F})$ and $16 \mathrm{~S}(\mathrm{G}, \mathrm{H})$. No significant differences were observed between grain As categories in E,F,G, and $\mathrm{H}$.

a.

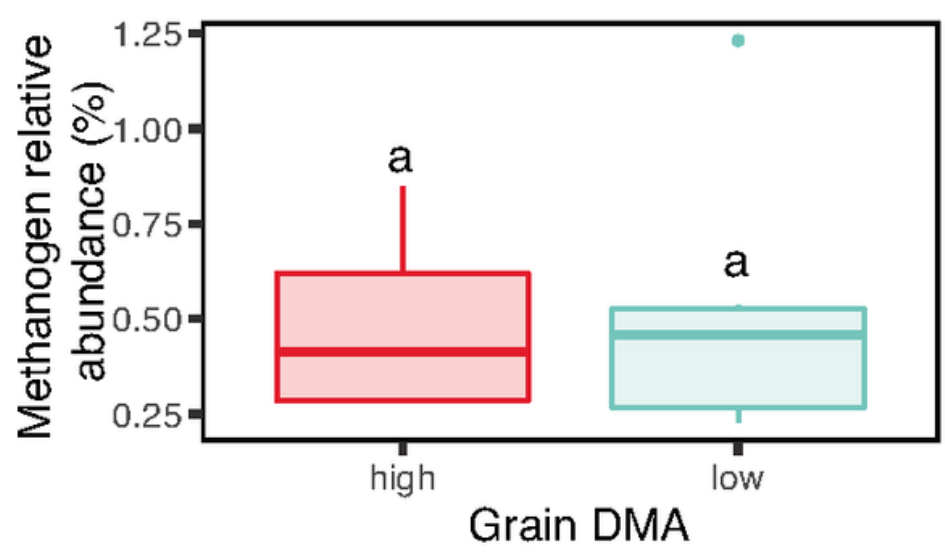

c.

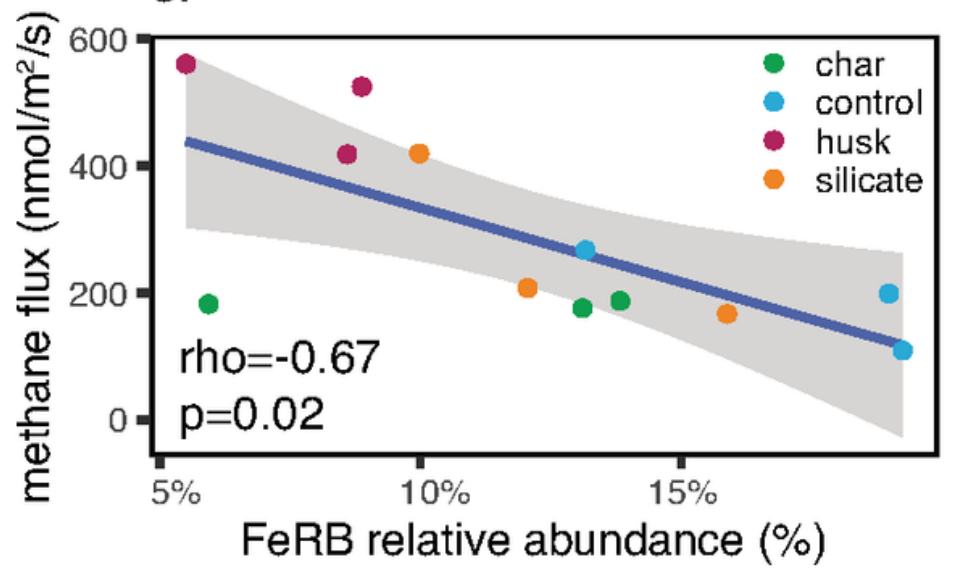

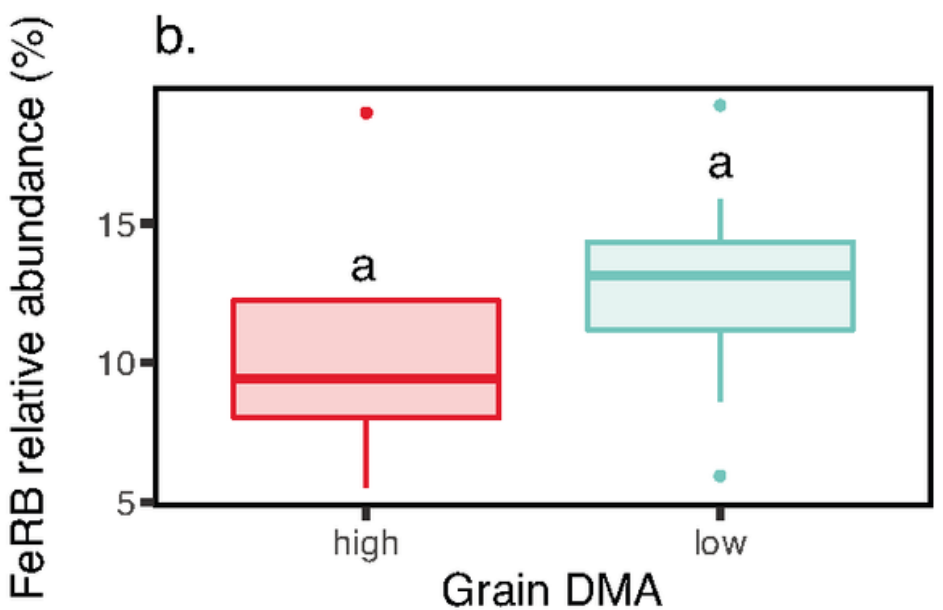

d.

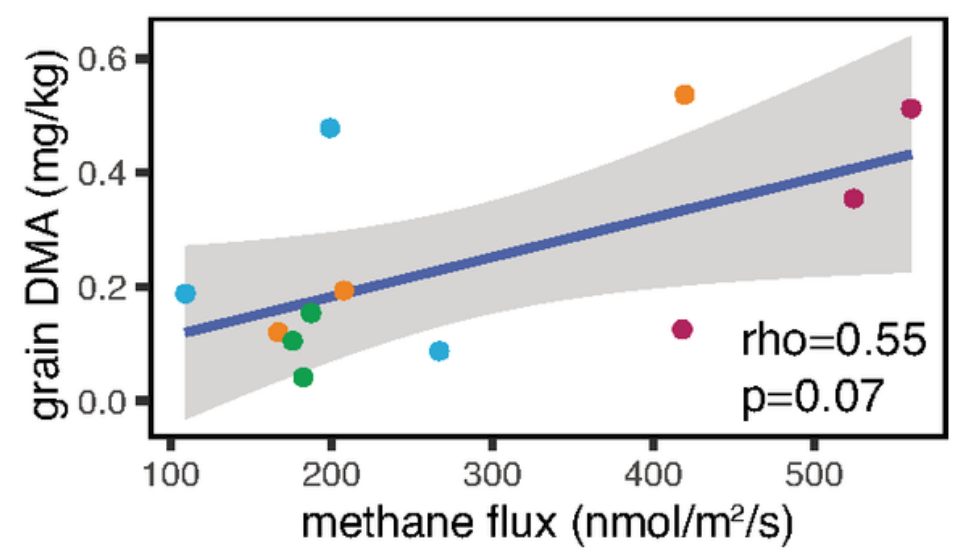

\section{Figure 11}

Boxplot of summed relative abundance of methanogens (A) and FeRB (B) in the rhizosphere at grain ripening compared across grain As categories, and correlations between median methane flux between 55-89 DPT and the summed relative abundance of FeRB (C), and grain DMA (D). In A and B no significant differences were observed across grain DMA categories. In $C$ and $D$, points are colored by paddy, blue line shows linear regression, and gray shaded region shows $95 \%$ confidence interval. 
a.

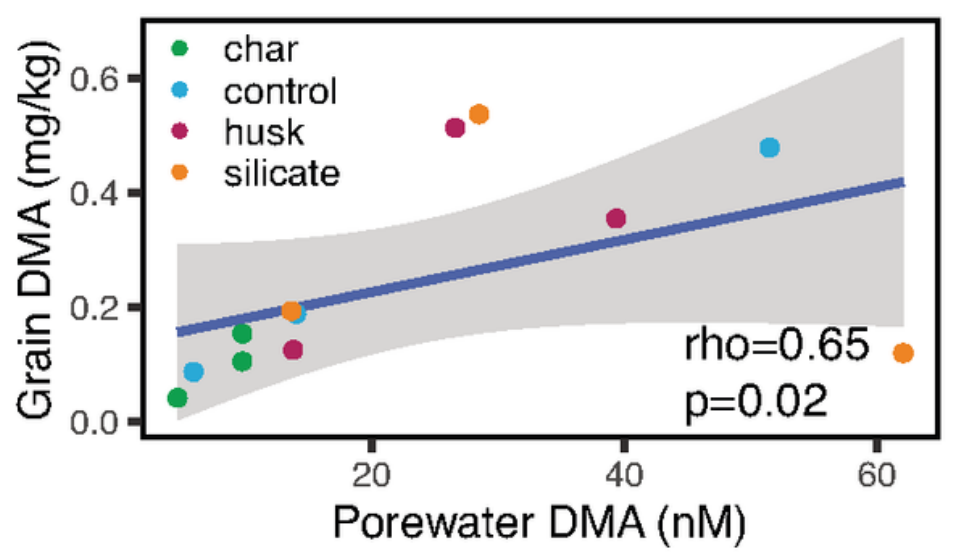

C.

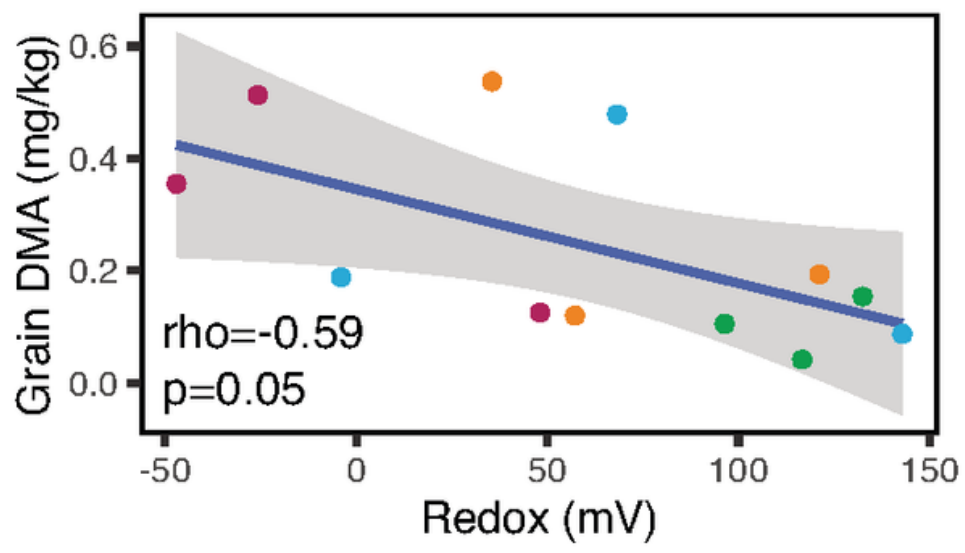

b.

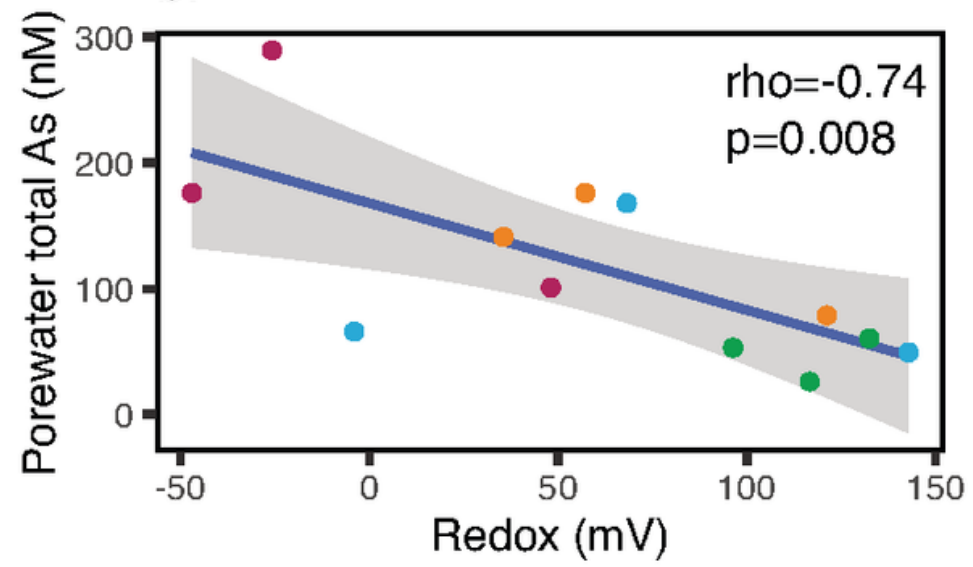

d.

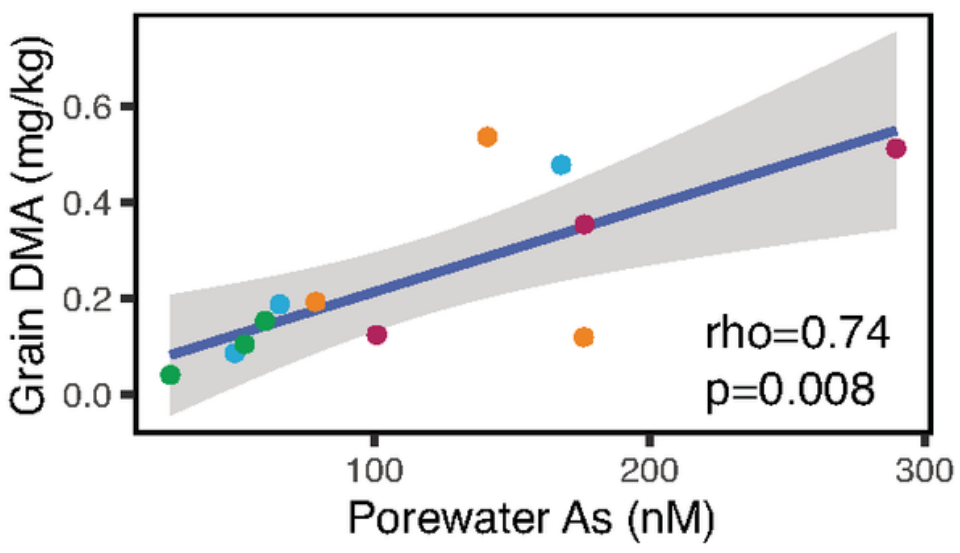

Figure 12

Correlations between median porewater chemistry related to grain organic As. A. grain DMA and median porewater DMA between 55 and 89 DPT. B. Median porewater As and median porewater redox between 55 and 89 DPT. C. Grain DMA and median redox between 55 and 89 DPT. D. Grain DMA and median porewater As between 55 and 89 DPT. Points are colored by paddy, blue line shows linear regression, gray shaded region shows $95 \%$ confidence interval.

\section{Supplementary Files}

This is a list of supplementary files associated with this preprint. Click to download.

- arsMseqSupplementaltables1Copy.xIsx

- arsMseqsupplementalinfo3.pdf 